\title{
Finite lattices and congruences. A survey
}

\author{
G. Grätzer And E. T. SChmidt
}

AbStract. In the early forties, R. P. Dilworth proved his famous result: Every finite distributive lattice $D$ can be represented as the congruence lattice of a finite lattice $L$. In one of our early papers, we presented the first published proof of this result; in fact we proved: Every finite distributive lattice $D$ can be represented as the congruence lattice of a finite sectionally complemented lattice $L$..

We have been publishing papers on this topic for 45 years. In this survey paper, we are going to review some of our results and a host of related results by others: Making $L$ "nice".

If being "nice" is an algebraic property such as being semimodular or sectionally complemented, then we have tried in many instances to prove a stronger form of these results by verifying that every finite lattice has a congruence-preserving extension that is "nice". We shall discuss some of the techniques we use to construct "nice" lattices and "nice" congruence-preserving extensions.

We shall describe some results on the spectrum of a congruence of a finite sectionally complemented lattice, measuring the sizes of the congruence classes. With very few restrictions, these can be as bad as we wish.

We shall also review some results on simultaneous representation of two distributive lattices. We conclude with the "magic wand" construction, which holds out the promise of obtaining results that go beyond what can be achieved with the older techniques.

Date: November 4, 2002.

2000 Mathematics Subject Classification: Primary: 06B10; Secondary: 06B15.

Key words and phrases: Congruence lattice, congruence-preserving extension, isoform, uniform, regular.

The research of the first author was supported by the NSERC of Canada.

The research of the second author was supported by the Hungarian National Foundation for Scientific Research, under Grant No. T29525. 


\section{Contents}

1. Introduction 3

1.1. Notation 3

2. Sectionally complemented lattices 3

2.1. Congruence-preserving extensions 3

2.2. Chopped lattices 4

2.3. The representation theorem 5

2.4. Rectangular extensions $\quad 7$

2.5. Constructing congruence-preserving extensions 8

3. Minimal representations $\quad 8$

3.1. The minimal construction $\quad 8$

3.2. Proving minimality 10

4. Semimodular lattices $\quad 12$

4.1. The representation theorem 12

4.2. Congruence-preserving extensions $\quad 13$

5. Independence theorems 14

6. Modular lattices $\quad 15$

6.1. The $M_{3}[D]$ construction $\quad 15$

6.2. Representation theorems 16

$\begin{array}{ll}\text { 6.3. Independence theorem } & 16\end{array}$

$\begin{array}{ll}\text { 7. Regular lattices } & 17\end{array}$

$\begin{array}{ll}\text { 7.1. The results } & 17\end{array}$

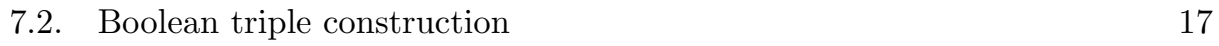

8. Uniform and isoform lattices 17

$\begin{array}{ll}\text { 8.1. Uniform lattices } & 17\end{array}$

8.2. Isoform lattices 18

9. Congruence class sizes in sectionally complemented lattices $\quad 19$

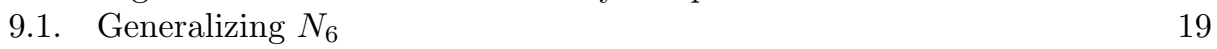

9.2. Spectrum 20

9.3. Valuation 21

10. Simultaneous representations of two distributive lattices 22

10.1. Restrictions as lattice homomorphisms 22

10.2. Extensions as join-homomorphisms 24

10.3. Huhn's result 24

10.4. Fixing the base lattice 25

10.5. Isotone maps 25

10.6. Huhn's theorem revisited 26

11. Beyond congruence-preserving extensions: Magic wands 27

11.1. The two magic wand theorems 27

$\begin{array}{ll}\text { 11.2. The construction } & 29\end{array}$ 


\section{Introduction}

The congruence lattice, Con $L$, of a finite lattice $L$ is a finite distributive lattice according to a result of N. Funayama and T. Nakayama [14]. The converse is a result of R.P. Dilworth from 1944 (see [6]): Every finite distributive lattice D can be represented as the congruence lattice, Con $L$, of a finite lattice $L$. This result was first published in 1962 in the paper [34] of the present authors.

In the last 60 years a large number of papers have been published strengthening and generalizing this result of Dilworth. These papers spilt into two categories:

(i) Finite congruence lattices.

(ii) Infinite congruence lattices.

The two fields are quite different. A finite distributive lattice is determined by the poset $\mathrm{J}(D)$ of join-irreducible elements. So a representation of a finite distributive lattice $D$ as the congruence lattice of a lattice $L$ is really a representation of a finite poset $P(=\mathrm{J}(D))$ as the poset of join-irreducible congruences of a finite lattice $L$. Now the join-irreducible congruences of a finite lattice $L$ are exactly the congruences of the form $\Theta(a, b)$, where $a \prec b$ in $L$, that is, $[a, b]$ is a prime interval. So it is enough to concentrate on such congruences, and make sure that they are partially ordered as required by $P$.

The infinite case is much different. We do not even have a characterization theorem: We do not know whether congruence lattices can be characterized as distributive algebraic lattices - as conjectured by Dilworth. A survey paper on the second field has just been written by J. Tůma and F. Wehrung [78]. In this survey, we stick with finite distributive lattices $D$ and (with a few exceptions) finite lattices $L$.

There are two types of representation theorems in this survey:

(1) The straight representation theorems.

(2) The congruence-preserving extension results.

We start this survey by illustrating these two types of theorems - and the major techniques used to prove them-with sectionally complemented lattices.

1.1. Notation. We use the standard notation, as in [16]. The $n$-element chain is denoted by $C_{n}$. A lattice is bounded, if it has zero, 0, and unit, 1 . For the bounded lattice $K$ and $L$, and homomorphism $\varphi: K \rightarrow L$, we call $\varphi$ a $\{0,1\}$ preserving homomorphism, if it map the zero of $K$ into the zero of $L$ and the unit of $K$ into the unit of $L$. We shall also use $\{0,1\}$-preserving meet-homomorphism, $\{0,1\}$-preserving join-homomorphism, 0-preserving meet-homomorphism, and 0preserving join-homomorphism with the obvious meanings.

\section{Sectionally complemented lattices}

2.1. Congruence-preserving extensions. First, we need our most important concept (G. Grätzer and E. T. Schmidt [38]): 


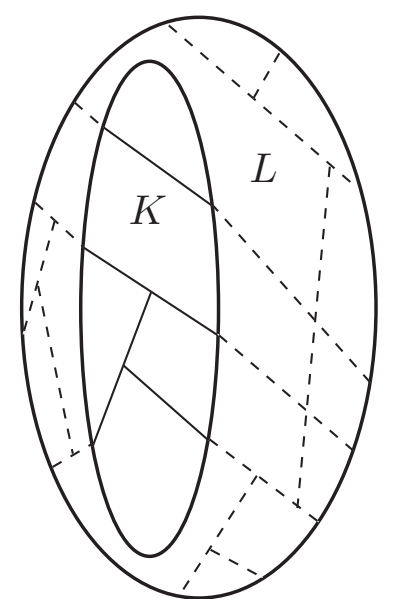

FiguRE 1. Illustrating a congruence-preserving extension.

Let $K$ be a finite lattice. A finite lattice $L$ is a congruence-preserving extension of $K$, if $L$ is an extension and every congruence of $K$ has exactly one extension to $L$. Of course, then the congruence lattice of $K$ is isomorphic to the congruence lattice of $L$. We could say that the congruence lattice of $K$ is naturally isomorphic to the congruence lattice of $L$ or that the algebraic reasons determining the congruence lattice of $K$ are carried over to $L$. For instance, let $a_{i}, b_{i} \in K, i=1,2,3$, and let

$$
\Theta\left(a_{1}, b_{1}\right) \vee \Theta\left(a_{2}, b_{2}\right)=\Theta\left(a_{3}, b_{3}\right)
$$

in $K$. Then $K$ has a finite partial sublattice $H$ forcing this equation. Since $L$ is an extension of $K$, it follows that $H$ is a partial sublattice of $L$, hence this equation holds also in $L$; and it holds for the same "algebraic reason" - namely, the existence of $H$.

And here is a pair of results (G. Grätzer and E. T. Schmidt [34] and [41]) illustrating the two types of representation theorems:

Theorem 1. Every finite distributive lattice D can be represented as the congruence lattice of a finite sectionally complemented lattice $L$.

Theorem 2. Every finite lattice $K$ has a finite, sectionally complemented, congruence-preserving extension $L$.

We are going to discuss in detail these results, and direct these discussions to demonstrate the main technical tools available to us.

2.2. Chopped lattices. We start with the most important lattice construction in this field.

Let $M$ be a poset satisfying the following two condition:

(1) $\inf \{a, b\}$ exists in $M$, for any $a, b \in M$;

(2) $\sup \{a, b\}$ exists for any $a, b \in M$ having a common upper bound in $M$. 
We define in $M$ :

$$
a \wedge b=\inf \{a, b\},
$$

and

$$
a \vee b=\sup \{a, b\},
$$

whenever $\sup \{a, b\}$ exists in $M$. This makes $M$ into a partial lattice, called a chopped lattice.

Now we define congruences and ideals of chopped lattices, as it is done for lattices.

An equivalence relation $\Theta$ of a chopped lattice $M$ is a congruence relation iff $a_{0} \equiv b_{0}(\Theta)$ and $a_{1} \equiv b_{1}(\Theta)$ imply that $a_{0} \wedge a_{1} \equiv b_{0} \wedge b_{1}(\Theta)$ and $a_{0} \vee a_{1} \equiv b_{0} \vee b_{1}$ $(\Theta)$, whenever $a_{0} \vee a_{1}$ and $b_{0} \vee b_{1}$ exist. The set Con $M$ of all congruence relations of $M$ partially ordered by set inclusion is again a lattice.

A subset $I$ of the chopped lattice $M$ is an ideal iff

(1) $i \in I$ and $a \in M$ imply that $a \wedge i \in I$;

(2) $i, j \in I$ implies that $i \vee j \in I$, provided that $i \vee j$ exists in $M$.

The set Id $M$ of all ideals of $M$ partially ordered by set inclusion is a lattice.

Chopped lattices are useful because of the following result due to G. Grätzer and H. Lakser [21] (first published in G. Grätzer [15]):

Lemma 1. Let $M$ be a finite chopped lattice. Then, for every congruence relation $\Theta$, there exists exactly one congruence relation $\bar{\Theta}$ of $\operatorname{Id} M$ such that, for a, $b \in M$,

$$
(a] \equiv(b] \quad(\bar{\Theta}) \quad \text { iff } \quad a \equiv b \quad(\Theta) .
$$

This result is very significant. It means that to construct a finite lattice $L$ to represent a given finite distributive lattice $D$ as a congruence lattice, it is sufficient to construct a finite chopped lattice $M$, since Con $M \cong \operatorname{Con} \operatorname{Id} M=\operatorname{Con} L$, where $L=\operatorname{Id} M$.

2.3. The representation theorem. To prove Theorem 1 , let $D$ be a finite distributive lattice, and form the finite poset $P=\mathrm{J}(D)$.

Lemma 2. Let $D$ be a finite distributive lattice. Then there exists a finite chopped lattice $M$ such that Con $M$ is isomorphic to $D$.

Let us illustrate this with $D$ the four-element chain; so $\mathrm{J}(D)$ is the three element chain $c \prec b \prec a$.

Take the finite set $M_{0}=\mathrm{J}(D) \cup\{0\}$ and make it a meet-semilattice by defining $\inf \{x, y\}=0$, if $x \neq y$. Note that the congruence relations of $M_{0}$ are in one-to-one

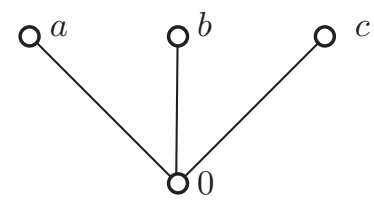

Figure 2. The chopped lattice $M_{0}$. 


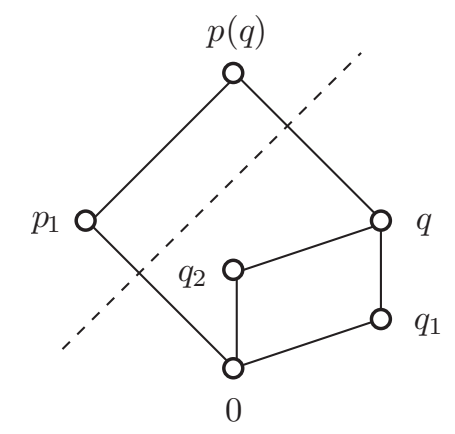

Figure 3 . The lattice $N_{6}=N(p, q)$.

correspondence with subsets of $\mathrm{J}(K)$. Thus Con $M_{0}$ is a Boolean lattice, whose atoms are associated with elements of $\mathrm{J}(K)$.

We must force that $a \equiv 0$ implies that $b \equiv 0$ implies that $c \equiv 0$.

To accomplish this, we use the lattice $N_{6}=N(p, q)$ of Figure 3 . Note that $N(p, q)$ has three congruence relations, namely, $\omega, \iota$, and $\Theta$, where $\Theta$ is the congruence relation with congruence classes $\left\{0, q_{1}, q_{2}, q\right\}$ and $\left\{p_{1}, p(q)\right\}$. Thus $\Theta\left(p_{1}, 0\right)=\iota$. In other words, $p_{1} \equiv 0$ "implies" that $q_{1} \equiv 0$, but $q_{1} \equiv 0$ "does not imply" that $p_{1} \equiv 0$.

We construct the finite chopped lattice $M$ by "inserting" $N(p, q)$ in $M_{0}$, for $a, b$ and for $b, c$, by appropriately doubling $b$ and $c$, see Figure 4 .

We are left with proving that $L=\operatorname{Id} M$ is sectionally complemented. Unfortunately, we cannot do better than refer the reader to G. Grätzer and E. T. Schmidt [34]. The proof is short but nontrivial. We have tried several times to prove something more general, but we have always failed. So let us raise this as a problem:

Problem 1. Let $M$ be a sectionally complemented chopped lattice. Under what conditions is Id $M$ sectionally complemented?

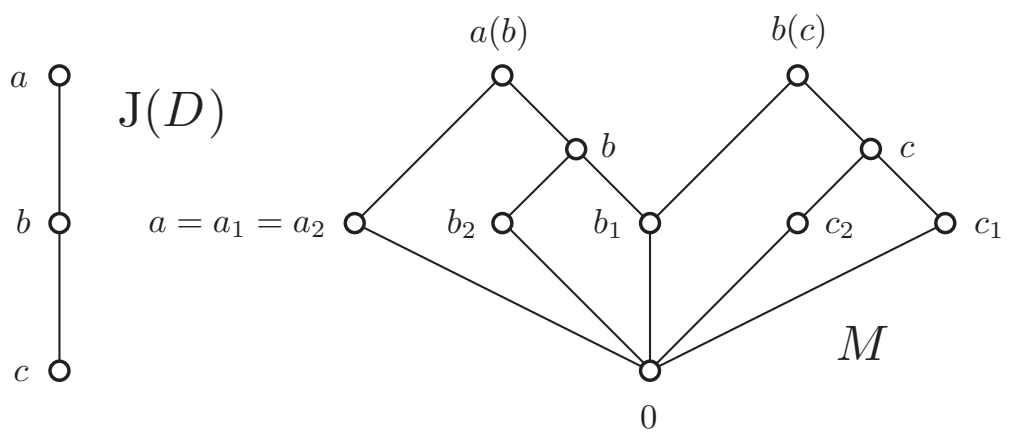

Figure 4 . The chopped lattice $M$. 
For a natural number $n$ and a class $\mathbf{V}$ of lattices, define $\operatorname{mcr}(n, \mathbf{V})$ as the smallest integer such that, for any distributive lattice $D$ with $n$ join-irreducible elements, there exists a finite lattice $L \in \mathbf{V}$ satisfying $\operatorname{Con} L \cong D$ and $|L| \leq \operatorname{mcr}(n, \mathbf{V})$.

Let SecComp denote the class of sectionally complemented lattices.

Problem 2. Determine $\operatorname{mcr}(n, \operatorname{SecComp})$. Is it $O\left(2^{2 n}\right)$ ?

The congruence lattice of a finite relatively complemented lattice is Boolean. So we cannot ask whether SecComp can be narrowed to the class of relatively complemented lattices. However, SecComp $\cap$ DuallySecComp would be a logical candidate. Note, however, that by Theorem 11 of our paper [34] and Lemma 4.16 of M. F. Janowitz [62], every finite lattice in SecComp $\cap$ DuallySecComp has a Boolean congruence lattice (see also [16], Theorem II.4.9).

Similarly, one can ask whether SecComp can be narrowed to the class of semimodular sectionally complemented lattices. The discussion in Section IV.3 of [16] (in particular, the top paragraph of p. 240) shows that this cannot be done either.

2.4. Rectangular extensions. Now we introduce the second major technique. The rectangular extension $\mathbb{R}(K)$ of a finite lattice $K$ is defined as the direct product of all subdirect factors of $K$, that is,

$$
\mathbb{R}(K)=\prod(K / \Phi \mid \Phi \in \mathrm{M}(\operatorname{Con} K)),
$$

where $\mathrm{M}(\mathrm{Con} K)$ is the set of all meet-irreducible congruences of $K$.

$K$ has a natural (diagonal) embedding into $\mathbb{R}(K)$ by

$$
\psi: a \mapsto a^{\mathbb{R}}=\langle[a] \Phi \mid \Phi \in \mathrm{M}(\operatorname{Con} K)\rangle .
$$

Let $K \psi=K^{\mathbb{R}}$ and for a congruence $\Theta$ of $K$, let $\Theta^{\mathbb{R}}$ denote the corresponding congruence of $K^{\mathbb{R}}$, that is, $\Theta^{\mathbb{R}}=\Theta \psi$.

Lemma 3. Let $K$ be a finite lattice. Then $K^{\mathbb{R}}$ has the Congruence Extension Property in $\mathbb{R}(K)$, that is, every congruence $\Theta$ of $K^{\mathbb{R}}$ has an extension $\bar{\Theta}$ to $\mathbb{R}(K)$, in formula, $\left.\bar{\Theta}\right|_{K^{\mathbb{R}}}=\Theta$.

For each $\Phi \in \operatorname{Id} M(\operatorname{Con} K)$, we select $S(K / \Phi)$, a finite, simple, sectionally complemented extension of $K / \Phi$. (We prove directly in G. Grätzer and E. T. Schmidt [41] that there is such an extension, but this also follows from much deeper results in the literature: O. Ore [64] and P. Pudlák and J. Tůma [66].)

Now we form the extension

$$
\widehat{\mathbb{R}}(K)=\prod(S(K / \Phi) \mid \Phi \in \mathrm{M}(\operatorname{Con} K))
$$

of $\mathbb{R}(K)$.

The extension $\widehat{\mathbb{R}}(K)$ of $K \cong K^{\mathbb{R}}$ has the following properties:

(1) $\widehat{\mathbb{R}}(K)$ is sectionally complemented.

(2) $\widehat{\mathbb{R}}(K)$ is finite.

(3) Select an atom $p_{\Phi}$ of $S(K / \Phi) \subseteq \widehat{\mathbb{R}}(K)$. Let

$$
P=\left\{p_{\Phi} \mid \Phi \in \mathrm{M}(\operatorname{Con} K)\right\} .
$$


Then there is a one-to-one correspondence between subsets of $P$ and congruences $\Theta$ of $\widehat{\mathbb{R}}(K)$; the subset of $P$ corresponding to the congruence $\Theta$ of $\widehat{\mathbb{R}}(K)$ is

$$
\left\{p_{\Phi} \mid p_{\Phi} \equiv 0(\Theta)\right\}
$$

hence, the congruence lattice of $\widehat{\mathbb{R}}(K)$ is a finite Boolean lattice.

(4) Every congruence $\Theta$ of $K$ has an extension $\widehat{\Theta}$ to a congruence of $\widehat{\mathbb{R}}(K)$ corresponding to the subset

$$
\left\{p_{\Phi} \mid \Phi \in \mathrm{M}(\operatorname{Con} K) \text { and } \Theta \not \leq \Phi\right\}
$$

of $P$.

2.5. Constructing congruence-preserving extensions. This construction is based on rectangular extensions and on the following statement (G. Grätzer and E. T. Schmidt [41]):

Lemma 4. Let $A$ and $B$ be (disjoint) finite sectionally complemented lattices with zero elements $0_{A}$ and $0_{B}$, respectively. Let $p_{A}$ be an atom of $A$ and let $p_{B}$ be an atom of $B$.

Form the partial lattice $M$ by identifying $0_{A}$ with $0_{B}$ and $p_{A}$ with $p_{B}$, respectively. Then $M$ is a finite chopped lattice and $\operatorname{Id} M$ is a finite sectionally complemented lattice.

The following statement is an easy consequence of Lemma 4:

Lemma 5. Let $A, B$, and $M$ be as in Lemma 4. In addition, let us assume that $B$ is a simple lattice. Then $\operatorname{Con} M \cong \operatorname{Con} A$ and $\operatorname{Id} M$ is a sectionally complemented congruence-preserving extension of $A$.

Now we can prove Theorem 2 by induction on $|\mathrm{J}(D)|$. We use chopped lattices repeatedly and the rectangular extension once; no other techniques are needed.

Problem 3. What is the size of the lattice $L$ we construct for Theorem 2? What is the minimum size of a lattice L satisfying Theorem 2?

\section{Minimal representations}

3.1. The minimal construction. Next, we take up the question of representations of small size for two reasons: This is one of the oldest problems in the field and the technique developed for its solution has other applications.

For a natural number $n$, define $\operatorname{mcr}(n)$ (minimal congruence representation) as the smallest integer such that, for any distributive lattice $D$ with $n$ join-irreducible elements, there exists a finite lattice $L$ satisfying $\operatorname{Con} L \cong D$ and $|L| \leq \operatorname{mcr}(n)$. Note that as defined in Section 2.3, $\operatorname{mcr}(n)=\operatorname{mcr}(n, \mathbf{L})$, where $\mathbf{L}$ is the class of all lattices.

The lattice $L$ constructed by R. P. Dilworth (see [6]) and the present authors [34] to represent $D$ is very large, it has $O\left(2^{2 n}\right)$ elements. This was improved to $O\left(n^{3}\right)$ in G. Grätzer and H. Lakser [22]; it was conjectured that $O\left(n^{3}\right)$ can be improved to $O\left(n^{2}\right)$ and that $O\left(n^{2}\right)$ is best possible. Indeed, $O\left(n^{2}\right)$ is possible, as proved in G. Grätzer, H. Lakser, and E. T. Schmidt [24]. 


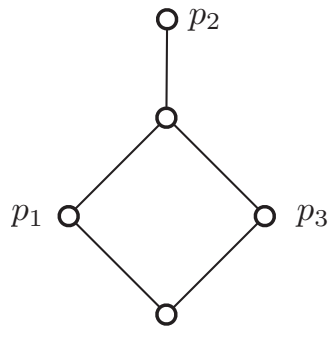

$D$

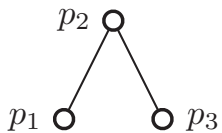

$P$

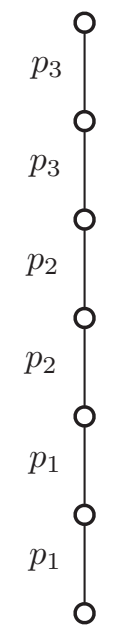

C

Figure 5. $D, P$, and $C$.

Theorem 3. Let $D$ be a finite distributive lattice with $n$ join-irreducible elements. Then there exists a planar lattice $L$ of $O\left(n^{2}\right)$ elements with Con $L \cong D$.

We illustrate the proof of this result with the distributive lattice $D$ of Figure 5 . The diagram also shows $P=\mathrm{J}(D)$ and the chain $C$ we form from $P$. The chain $C$ is of length $2|P|=6$, and the prime intervals are "marked" with elements of $P$ as illustrated. We call this "marking" coloring.

Figure 6 shows the building blocks we are going to use: $M_{3}$ and $N_{5,5}$. Now we proceed as follows: To construct $L$, we take $C^{2}$. If both lower edges of a covering square in $C^{2}$ have the same color, we add an element to make it a covering $M_{3}$.
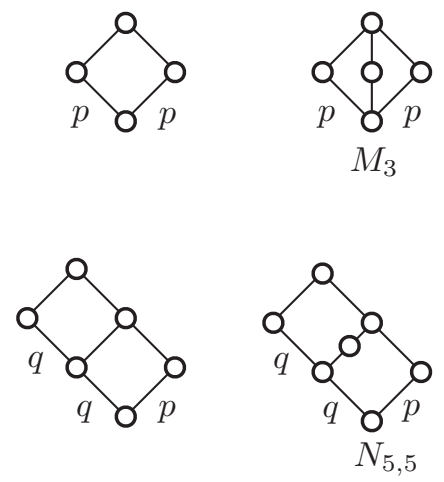

Figure 6 . The two building blocks, for $p<q$. 


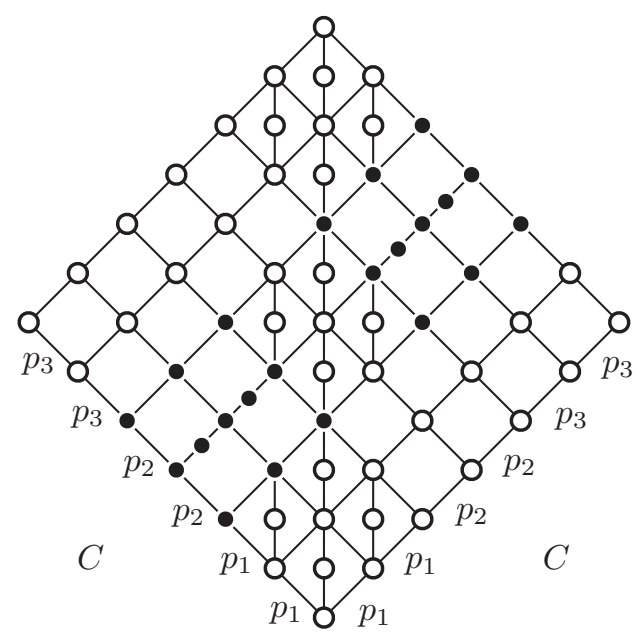

Figure 7. The minimal lattice $L$.

If in $C^{2}$ we have a covering $C_{2} \times C_{3}$, where the lower $C_{2}$ is colored by $p$, the lower $C_{3}$ is colored by $q$ twice, where $p<q$, then we add an element to make it an $N_{5,5}$.

The lattice $L$ we obtain is shown in Figure 7 . (The copies of $N_{5,5}$ in the diagram are marked by black-filled elements.)

It is an easy computation to show that, in general, $|L| \leq k n^{2}$, for some constant $k$, and that $D \cong$ Con $L$; this isomorphism is established by assigning to $p \in P$ the congruence of $L$ generated by collapsing any (all) prime intervals of color $p$.

A somewhat sharper result is the following:

Theorem 4. For any integer $n \geq 2$,

$$
\frac{1}{16} \frac{n^{2}}{\log _{2} n}<\operatorname{mcr}(n)<3(n+1)^{2} .
$$

The upper bound was proved in G. Grätzer, H. Lakser, and E. T. Schmidt [24] and the lower bound in G. Grätzer and D. Wang [51].

Y. Zhang [80] noticed that the proof of this inequality can be improved to obtain the following result: for $n \geq 64$,

$$
\frac{1}{64} \frac{n^{2}}{\left(\log _{2} n\right)^{2}}<\operatorname{mcr}(n) .
$$

The lower bound $\frac{n^{2}}{16 \log _{2} n}$ for $\operatorname{mcr}(n)$ is weaker than the last one.

3.2. Proving minimality. The real combinatorics is in G. Grätzer, I. Rival, and N. Zaguia [33], proving that $O\left(n^{2}\right)$ is, indeed, best possible.

Theorem 5. Let $\alpha$ be a real number satisfying the following condition: Every distributive lattice $D$ with $n$ join-irreducible elements can be represented as the congruence lattice of a lattice $L$ with $O\left(n^{\alpha}\right)$ elements. Then $\alpha \geq 2$. 


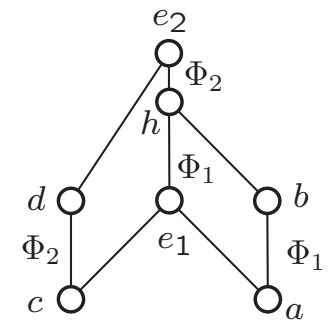

Figure 8. The crucial weak projectivities.

We outline the idea of the proof. Let $n$ be a natural number. Let $D_{n}$ be a distributive lattice whose partially ordered set $P_{n}$ of join-irreducible elements is bipartite (that is, every element is either maximal or minimal) and in which there are $O\left(n^{2}\right)$ covering edges. Let $L_{n}$ be a lattice satisfying Con $L_{n} \cong D_{n}$, and contrary to the theorem assume that $L_{n}$ has $O\left(n^{\alpha}\right)$ elements, where $\alpha<2$.

There are $O\left(n^{2}\right)$ covering pairs of join-irreducible congruences of $L_{n}$. We can associate with each such pair $\Phi_{1} \prec \Phi_{2}$ a three-element chain $e_{1}<h<e_{2}$ in $L_{n}$ such that $\Phi_{1}=\Theta\left(e_{1}, h\right)$ and $\Phi_{2}=\Theta\left(e_{2}, h\right)$. Indeed, let $\Phi_{1}=\Theta(a, b)$ and $\Phi_{2}=\Theta(c, d)$, with $a \prec b$ and $c \prec d$ in $L_{n}$. Since $\Phi_{1} \leq \Phi_{2}$, there is a sequence of weak projectivities from $c / d$ into $a / b$. Choosing $a / b$ and $c / d$ as close to each other as we can, we obtain the situation depicted in Figure 8.

Since there are $O\left(n^{2}\right)$ covering pairs of join-irreducible congruences and there are $O\left(n^{\alpha}\right)$ elements in $L_{n}$, there must be an element $h \in L_{n}$ that appears as the middle element of $O\left(n^{2} / n^{\alpha}\right)=O\left(n^{2-\alpha}\right)$ three-element chains. For half of these chains, still $O\left(n^{2-\alpha}\right)$ in number, the top interval (or dually, the bottom interval) defines the larger congruence. So we obtain in $L_{n}$ an element $h$ and a set $A$ of elements, $O\left(n^{2-\alpha}\right)$ in number, so that all the $\Theta(h, x)$ are maximal (or all are minimal) join-irreducible congruences of $L_{n}$. Obviously, these congruences are pairwise incomparable. It is easy to verify that $A$ - under join-generates a free join-semilattice $F$ (or in the dual case, a free meet-semilattice). The set $F \subseteq L_{n}$ has $O\left(2^{n^{2-\alpha}}\right)$ elements. But this is a contradiction since $L_{n}$ has only $O\left(n^{2}\right)$ elements.

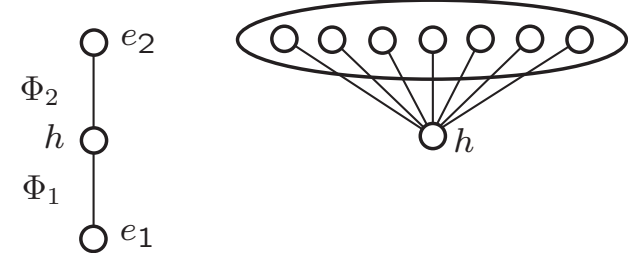

Figure 9. Constructing the set $A$. 

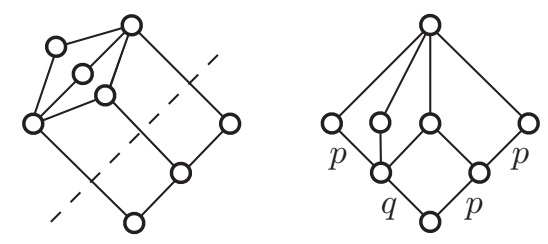

Figure 10. Two views of the building block $S_{8}$, for $p<q$.

A different kind of lower bound is obtained in R. Freese [10]; it is shown that if $\mathrm{J}(\operatorname{Con} L)$ has $e$ edges $(e>2)$, then

$$
\frac{e}{2 \log _{2} e} \leq|L| .
$$

Freese also proves that $\mathrm{J}(\mathrm{Con} L)$ can be computed in time $O\left(|L|^{2} \log _{2}|L|\right)$.

Consider the optimal length of $L$. E. T. Schmidt [70] constructs a finite lattice $L$ of length $5 m$, where $m$ is the number of dual atoms of $D$ (for finite chains, this was done in J. Berman [4]); S.-K. Teo [74] proves that this result is best possible.

Problem 4. In Theorem 5, is the construction "best" is some sharper sense?

\section{Semimodular lattices}

4.1. The representation theorem. G. Grätzer, H. Lakser, and E. T. Schmidt [27] provide for semimodular lattices a very good representation theorem:

Theorem 6. Every finite distributive lattice $D$ can be represented as the congruence lattice of a finite semimodular lattice $S$. In fact, $S$ can be constructed as a planar lattice of size $O\left(n^{3}\right)$, where $n$ is the number of join-irreducible elements of $D$.

The proof of this result is very similar to the proof of Theorem 3. The basic building block is $S_{8}$; we show two views of this lattice in Figure 10: The first view shows $S_{8}$ with its only nontrivial congruence indicated with a dashed line; the second views shows $S_{8}$ as it is used in the construction.

To illustrate the construction, take the distributive lattice $D$ of Figure 11; the poset $\mathrm{J}(D)$ is also shown.
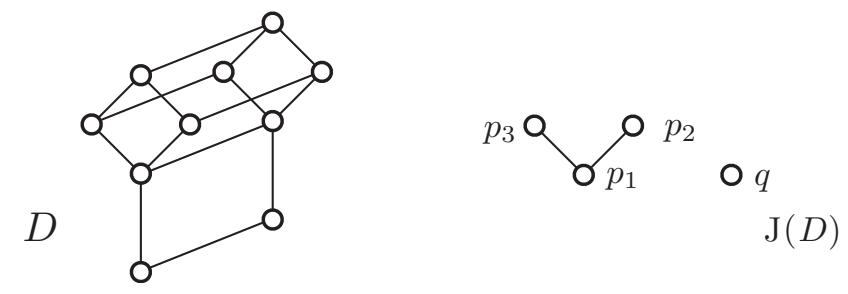

Figure 11. $D$ and $\mathrm{J}(D)$ 


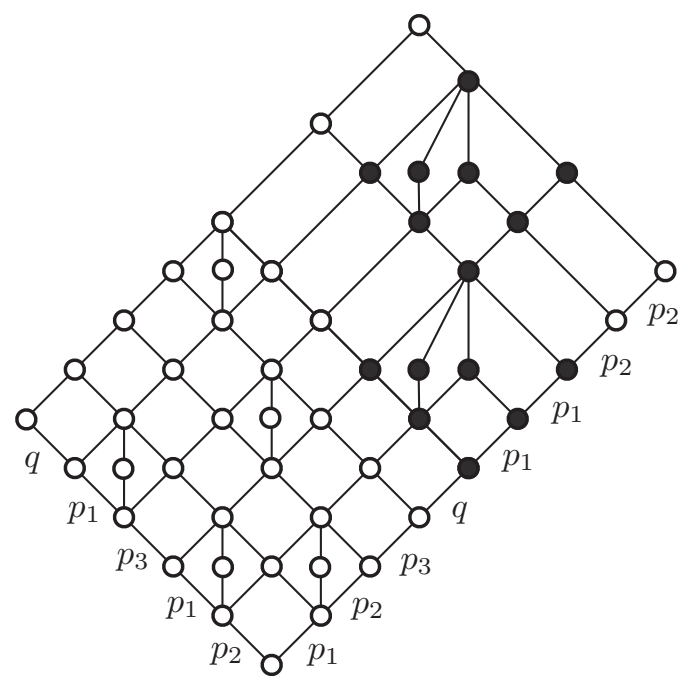

Figure 12. The lattice $S$.

Now we form two chains and their direct products, see Figure 12. For $p_{1} \prec p_{2}$, we replace the corresponding $C_{3} \times C_{3}$ by $S_{8}$. We make a similar replacement for $p_{1} \prec p_{3}$, to obtain $S$. (The two copies of $S_{8}$ in the diagram are marked by blackfilled elements.)

Let SemiMod and Planar + SemiMod denote the class of semimodular and planar semimodular lattices, respectively.

Problem 5. Is $\operatorname{mcr}(n, \operatorname{SemiMod})=\operatorname{mcr}(n$, Planar $+\operatorname{SemiMod})=O\left(n^{3}\right)$ ?

Recall that the function mor was defined in Section 2.3 .

4.2. Congruence-preserving extensions. We also found a congruence-preserving extension theorem for semimodular lattices in G. Grätzer and E. T. Schmidt [45]:

Theorem 7. Every finite lattice $K$ has a congruence-preserving embedding into a finite semimodular lattice $L$.

The proof starts out with the rectangular extension $\mathbb{R}(K)$ of $K$. Then we embed each direct factor of the rectangular extension into a simple semimodular lattice, and their direct product $\widehat{\mathbb{R}}(K)$ is a further extension of $K$. These embeddings of the direct factors we obtain as an application of a result of R.P. Dilworth, see [6] and [7]. The congruences then are represented in a dual ideal $F$ of $\widehat{\mathbb{R}}(K)$ that is Boolean. By gluing a modular lattice $M$ to $\widehat{\mathbb{R}}(K)$, we can make the join-irreducible congruences to be represented on a dual ideal $E$ of $M$ that is a chain, so the proof is completed by gluing the lattice $S$ (see Figure 12) to the construct, see Figure 13.

Problem 6. What is the size of the lattice $L$ we construct for Theorem 7? What is the minimum size of a lattice $L$ satisfying Theorem 7? 


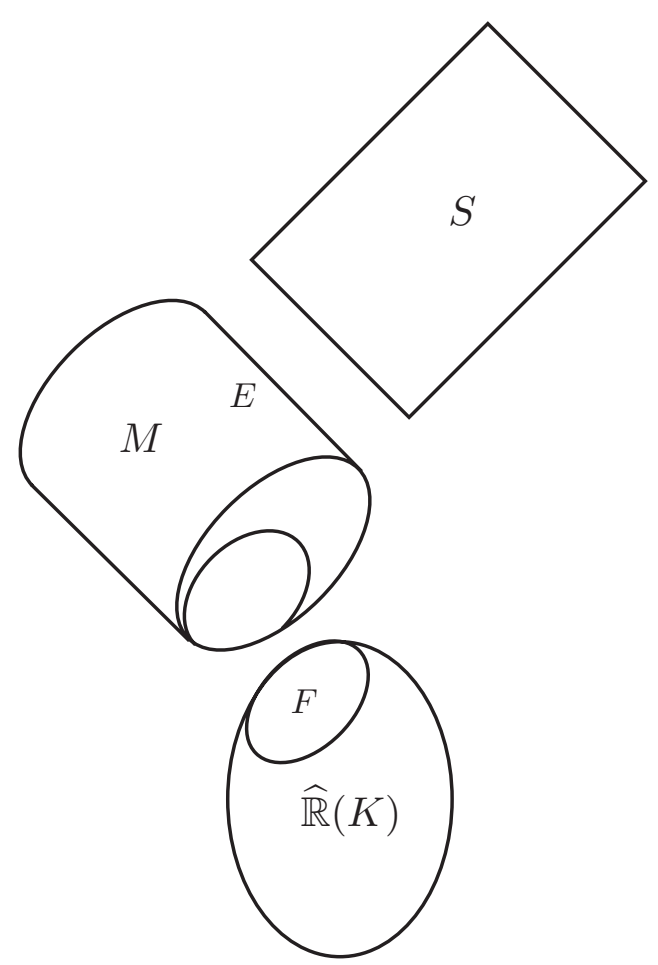

Figure 13. The final gluing.

\section{Independence theorems}

The following problem was first raised in the first edition of [16] (Problem II.18):

Problem. Let $K$ be a lattice with more than one element, and let $G$ be a group. Does there exist a lattice $L$ such that the congruence lattice of $L$ is isomorphic to the congruence lattice of $K$ and the automorphism group of $L$ is isomorphic to $G$ ? If $K$ and $G$ are finite, can $L$ chosen to be finite?

This problem was solved for finite lattices by V. A. Baranskiı [2], [3] and A. Urquhart [79]. We now state the Baranskiu-Urquhart theorem:

Theorem 8 (The Independence Theorem). Let $D$ be a finite distributive lattice with more than one element, and let $G$ be a finite group. Then there exists a finite lattice $L$ such that the congruence lattice of $L$ is isomorphic to $D$ and the automorphism group of $L$ is isomorphic to $G$.

This is a representation theorem; both published proofs rely heavily on the representation theorem of finite distributive lattices as congruence lattices of finite lattices (R.P. Dilworth) and on the representation theorem of finite groups as automorphism groups of finite lattices (G. Birkhoff [5]). 
There is also a congruence-preserving extension variant, which we published in G. Grätzer and E. T. Schmidt [38]:

Theorem 9 (The Strong Independence Theorem). Let $K$ be a finite lattice with more than one element and let $G$ be a finite group. Then $K$ has a congruence-preserving extension $L$ whose automorphism group is isomorphic to $G$.

There is a stronger form of this theorem; to state it, we need the analogue of the congruence-preserving extension concept for automorphisms.

Let $K$ be a lattice. $L$ is an automorphism-preserving extension of $K$, if $L$ is an extension and every automorphism of $K$ has exactly one extension to $L$; moreover, every automorphism of $L$ is the extension of an automorphism of $K$.

Of course, then the automorphism group of $K$ is isomorphic to the automorphism group of $L$.

Now we state the stronger version of Theorem 9:

Theorem 10 (The Strong Independence Theorem, Full Version). Let $K_{C}$ and $K_{A}$ be finite lattices with more than one element satisfying $K_{C} \cap K_{A}=\{0\}$. Then there exists a lattice $L$ such that the following conditions hold:

(1) $L$ is a finite, atomistic, 0-preserving extension of both $K_{A}$ and $K_{C}$.

(2) $L$ is a congruence-preserving extension of $K_{C}$.

(3) $L$ is an automorphism-preserving extension of $L_{A}$.

Of course, then the congruence lattice of $L$ is isomorphic to the congruence lattice of $K_{C}$, and the automorphism group of $L$ is isomorphic to the automorphism group of $K_{A}$.

Problem 7. What is the size of the lattice $L$ we construct for Theorem 9? What is the minimum size of a lattice $L$-as a function of $|K|$ and $|G|$-satisfying Theorem 9?

Problem 8. What is the size of the lattice $L$ we construct for Theorem 10? What is the minimum size of a lattice $L$-as a function of $\left|K_{C}\right|$ and $\left|K_{A}\right|$-satisfying Theorem 10?

\section{Modular lattices}

6.1. The $M_{3}[D]$ construction. Let $D$ be a bounded distributive lattice, and let $M_{3}=\{0, a, b, c, 1\}$ be the five-element nondistributive modular lattice. Let $M_{3}[D]$ denote the subposet of $D^{3}$ consisting of all $\langle x, y, z\rangle$ satisfying

$$
x \wedge y=y \wedge z=z \wedge x .
$$

We call such a triple balanced.

Then the following statements hold (E. T. Schmidt [67]):

(i) $M_{3}[D]$ is a modular lattice.

(ii) The subset

$$
\bar{M}_{3}=\{\langle 0,0,0\rangle,\langle 1,0,0\rangle,\langle 0,1,0\rangle,\langle 0,0,1\rangle,\langle 1,1,1\rangle\}
$$

of $M_{3}[D]$ is a sublattice of $M_{3}[D]$ and it is isomorphic to $M_{3}$. 


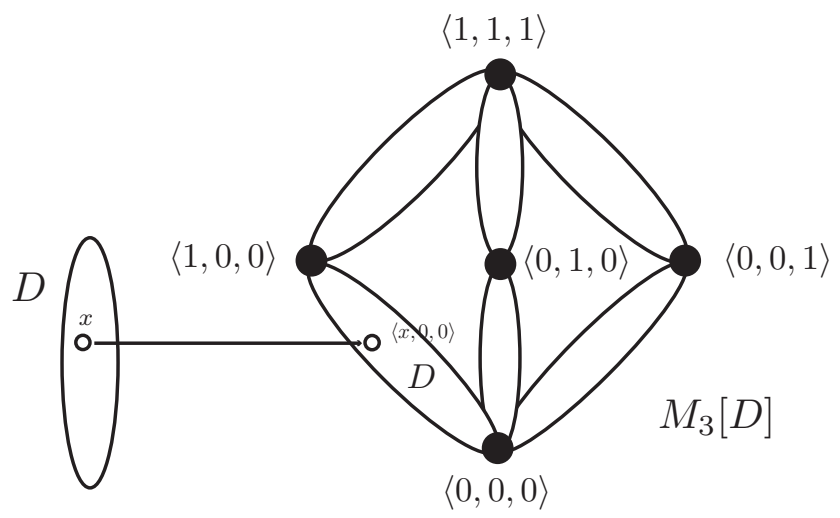

Figure 14. The $M_{3}[D]$ construction.

(iii) The subposet $\bar{D}=\{\langle x, 0,0\rangle \mid x \in D\}$ of $M_{3}[D]$ is isomorphic to $D$; we identify $D$ with $\bar{D}$.

(iv) $\bar{M}_{3}$ and $D$ generate $M_{3}[D]$.

(v) Let $\Theta$ be a congruence relation of $D=\bar{D}$; then there is a unique congruence $\bar{\Theta}$ of $M_{3}[D]$ such that $\bar{\Theta}$ restricted to $\bar{D}$ is $\Theta$; therefore, Con $M_{3}[D] \cong$ Con $D$.

See Figure 14. In today's terminology, $M_{3}[D]$ is a congruence-preserving extension of $D$.

6.2. Representation theorems. The congruence lattice of a finite modular lattice is Boolean; however, a finite distributive lattice has a representation as the congruence lattice of an infinite modular lattice by E. T. Schmidt [69]:

Theorem 11. Every finite distributive lattice $D$ can be represented as the congruence lattice of a modular lattice $M$.

For a shorter proof, see [73]. R. Freese [9] proved that $M$ can be constructed as a finitely generated modular lattice.

A much deeper result was proved in E. T. Schmidt [71]:

Theorem 12. Every finite distributive lattice $D$ can be represented as the congruence lattice of a complemented modular lattice $M$.

6.3. Independence theorem. In G. Grätzer and E. T. Schmidt [46], we prove the independence theorem for (infinite) modular lattices with finite congruence lattices.

Theorem 13 (Independence Theorem for Modular Lattices with Finite Congruence Lattices). Let $D$ be a finite distributive lattice with more than one element and let $G$ be a finite group. Then there exists a modular lattice $M$ such that the congruence lattice of $M$ is isomorphic to $D$ and the automorphism group of $M$ is isomorphic to $G$. 


\section{Regular lattices}

7.1. The results. Let $L$ be a lattice. We call a congruence relation $\Theta$ of $L$ regular, if any congruence class of $\Theta$ determines the congruence. Let us call the lattice $L$ regular, if all congruences of $L$ are regular.

Sectionally complemented lattices are regular, so we already have a representation theorem (Theorem 1) and a congruence-preserving extension version (Theorem 2). More generally,

Theorem 14. Every lattice $L$ has a congruence-preserving embedding into a regular lattice $\tilde{L}$.

7.2. Boolean triple construction. For a bounded lattice $K$, let us call the triple $\langle x, y, z\rangle \in L^{3}$ boolean iff the following equations hold:

$$
\begin{aligned}
& x=(x \vee y) \wedge(x \vee z), \\
& y=(y \vee x) \wedge(y \vee z), \\
& z=(z \vee x) \wedge(z \vee y) .
\end{aligned}
$$

Note that in a distributive lattice, a triple is balanced iff it is boolean. We denote by $\mathbf{M}_{3}\langle K\rangle \subseteq K^{3}$ the poset of all boolean triples of $K . \mathbf{M}_{3}\langle K\rangle$ is a bounded lattice. We identify the lattice $K$ with the interval $[\langle 0,0,0\rangle,\langle 1,0,0\rangle]$ under the isomorphism $x \mapsto\langle x, 0,0\rangle$.

Theorem 15 (G. Grätzer and F. Wehrung [52]). $\mathbf{M}_{3}\langle K\rangle$ is a congruence-preserving extension of $K$.

In fact, if $\mathbf{M}_{3}\langle K, a\rangle$ denotes the dual ideal of $\mathbf{M}_{3}\langle K\rangle$ generated by $\langle 0, a, 0\rangle(a \in$ $K)$, then the map $\varphi_{a}: x \mapsto\langle x, a, x \wedge a\rangle$ is a congruence-preserving embedding. Then for $b, c \in K, a<b$, the element $d=\langle c, b, c \wedge b\rangle \in \mathbf{M}_{3}\langle K, a\rangle$ satisfies $\Theta\left(a \varphi_{a}, b \varphi_{a}\right)=\Theta\left(c \varphi_{a}, d\right)$.

Since a lattice $L$ is regular iff for all $a<b$ and $c \in L$, there exists a $d \in L$ with $\Theta(a, b)=\Theta(c, d)$, Theorem 14 easily follows.

The Boolean triple construction is a special case of the lattice tensor product construction of G. Grätzer and F. Wehrung that provides - among other results - a congruence-preserving extension associated with every finite simple lattice; see the survey papers G. Grätzer and F. Wehrung [55] and J. Tůma and F. Wehrung [78].

\section{Uniform and isoform lattices}

8.1. Uniform lattices. Let $L$ be a lattice. We call a congruence relation $\Theta$ of $L$ uniform, if any two congruence classes of $\Theta$ are of the same size (cardinality). Let us call the lattice $L$ uniform, if all congruences of $L$ are uniform. The following result was proved in G. Grätzer, E. T. Schmidt, and K. Thomsen [50]:

Theorem 16. Every finite distributive lattice $D$ can be represented as the congruence lattice of a finite uniform lattice $L$.

A uniform lattice is always regular, so the lattices of Theorem 16 are also regular. Figure 15 shows the result of the construction for $D=C_{4}$. 


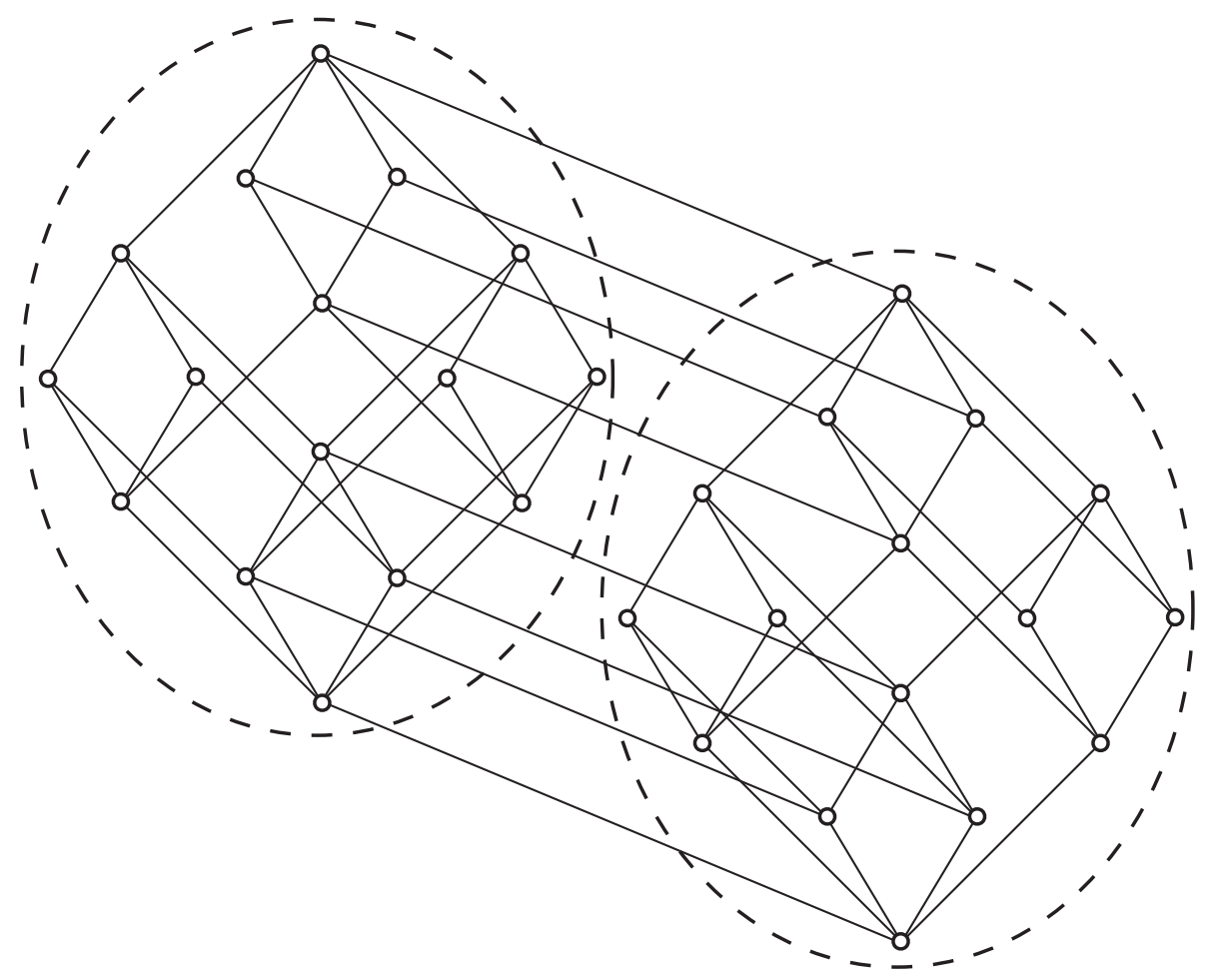

FigURE 15. The uniform construction for the four-element chain.

8.2. Isoform lattices. Let $L$ be a lattice. We call a congruence relation $\Theta$ of $L$ isoform, if any two congruence classes of $\Theta$ are isomorphic (as lattices). Let us call the lattice $L$ isoform, if all congruences of $L$ are isoform.

Theorem 17 (G. Grätzer and E. T. Schmidt [49]). Every finite distributive lattice $D$ can be represented as the congruence lattice of a finite isoform lattice $L$.

Since isomorphic lattices are of the same size, Theorem 17 is a stronger version of Theorem 16. Figure 15 shows that the lattice we obtained in G. Grätzer, E. T. Schmidt, and K. Thomsen [50] for $D=C_{4}$ is not isoform.

Let $P=\left\langle P ; \leq_{P}\right\rangle$ be a finite poset. Then the partial ordering $\leq_{P}$ on $P$ is the reflexive-transitive extension of $\prec_{P}$, the covering relation in $\left\langle P ; \leq_{P}\right\rangle$, in formula: $\operatorname{rt}\left(\prec_{P}\right)=\leq_{P}$. Now take a subset $H$ of $\prec_{P}$, and take the reflexive-transitive extension $\operatorname{rt}(H)$ of $H$. Then $\langle P ; \operatorname{rt}(H)\rangle$ is also a poset; we call it a pruning of $P$. If you think of $P$ in terms of its diagram, then the terminology is easy to picture: We obtain the diagram of $\langle P ; \operatorname{rt}(H)\rangle$ from the diagram of $P$ by cutting out (pruning) some edges (each representing a covering) but not deleting any elements. For instance, the lattice of Figure 15 is a pruning of the Boolean lattice $C_{2}^{5}$.

Here is a more complete version of Theorem 17: 
Theorem 18 (G. Grätzer and E. T. Schmidt [49]). Every finite distributive lattice $D$ can be represented as the congruence lattice of a finite lattice $L$ with the following properties:

(i) $L$ is isoform.

(ii) For every $\Theta$ of $L$, the congruence classes of $\Theta$ are projective intervals.

(iii) $L$ is a pruned finite Boolean lattice.

By Properties (i) and (ii), for every congruence relation $\Theta$ of $L$ and for any two congruence classes $U$ and $V$ of $\Theta$, the congruence classes $U$ and $V$ are required to be isomorphic and projective intervals, but we do not require that there be a projectivity that is also an isomorphism.

Problem 9. Does every finite lattice have a congruence-preserving extension into a finite uniform (isoform) lattice?

Problem 10. Are infinite relatively complemented lattices uniform?

Problem 11. Is it possible to sharpen Theorem 18 so that the isomorphisms of the congruence classes be projectivities?

Problem 12. Can every finite distributive lattice $D$ be represented as the congruence lattice of a finite uniform lattice $L$ with some additional property: $L$ be semimodular, relatively complemented, 2-distributive, and so on?

We also raise the question, what happens in the infinite case:

Problem 13. Is there an analogue of Theorem 17 for infinite lattices?

Let Uniform and Isoform denote the class of uniform and isoform lattices, respectively.

Problem 14. What is $\operatorname{mcr}(n$, Uniform)? What is $\operatorname{mcr}(n$, Isoform)?

\section{Congruence class sizes in sectionally complemented lattices}

9.1. Generalizing $N_{6}$. The basic building block of the lattice $L$ in Theorem 1 is the lattice $N_{6}$ of Figure 3. This lattice has some crucial properties:

(i) $N_{6}$ is sectionally complemented.

(ii) $N_{6}$ has exactly one nontrivial congruence $\Theta$.

(iii) $\Theta$ has exactly two congruence classes: the prime ideal $\left\{0, q_{1}, q_{2}, q\right\}$ and the dual prime ideal $\left\{p_{1}, p(q)\right\}$.

(iv) $p_{1} \equiv 0(\Phi)$ implies that $q_{1} \equiv 0(\Phi)$, for every congruence $\Phi$ of $N_{6}$.

We can associate with $\Theta$ the pair $\langle 4,2\rangle$ measuring the size of the two congruence classes. We start with the following question: Which pairs $\left\langle t_{1}, t_{2}\right\rangle$ can substitute for $\langle 4,2\rangle$ ? In other words, for which pairs of integers $\left\langle t_{1}, t_{2}\right\rangle$ is there a finite lattice $L$ such that

(1) $L$ is sectionally complemented.

(2) $L$ has exactly one nontrivial congruence $\Theta$.

(3) $\Theta$ has exactly two congruence classes: the prime ideal $P$ and the dual prime ideal $Q$ satisfying that $|P|=t_{1}$ and $|Q|=t_{2}$.

This question is answered as follows: 
Theorem 19 (G. Grätzer and E. T. Schmidt [48]). Let $\left\langle t_{1}, t_{2}\right\rangle$ be a pair of natural numbers. Then there is a finite lattice $L$ with properties (1)-(3) iff $\left\langle t_{1}, t_{2}\right\rangle$ satisfies the following three conditions:

$\left(P_{1}\right) 2 \leq t_{1}$ and $t_{1} \neq 3$.

$\left(P_{2}\right) 2 \leq t_{2}$ and $t_{2} \neq 3$.

$\left(P_{3}\right) t_{1}>t_{2}$.

Figure 16 illustrates the lattice we obtain for $\langle 5,4\rangle$.

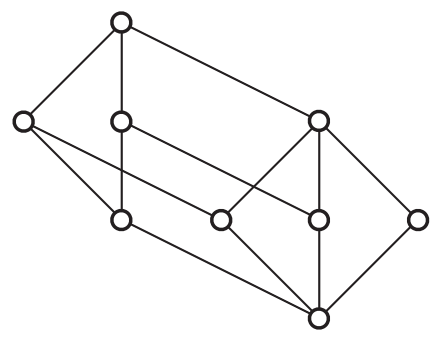

Figure 16. A lattice representing $\langle 5,4\rangle$.

9.2. Spectrum. The question answered by Theorem 19 is a very special case of a more general problem: What can we say about the cardinalities of the congruence classes of a nontrivial congruence in a finite sectionally complemented lattice?

Let $L$ be a finite lattice, and let $\Theta$ be a congruence of $L$. We denote by $\operatorname{Spec} \Theta$ the spectrum of $\Theta$, that is, the family of cardinalities of the congruence classes of $\Theta$. So Spec $\Theta$ has $|L / \Theta|$ elements, and each element is an integer $\geq 1$.

It is clear that if $S$ is a family of integers $\geq 1$, then it is the spectrum of some congruence (take $L$ as an appropriate chain). We are interested in the following problem: Characterize the spectra of nontrivial congruences of finite sectionally complemented lattices.

This problem is completely solved by the following result (G. Grätzer and E. T. Schmidt [48]):

Theorem 20. Let $S=\left(m_{j} \mid j<n\right)$ be a family of natural numbers, $n \geq 1$. Then there is a finite sectionally complemented lattice $L$ with more than one element and a nontrivial congruence $\Theta$ of $L$ such that $S$ is the spectrum of $\Theta$ iff $S$ satisfies the following conditions:

$\left(S_{1}\right) 2 \leq n$ and $n \neq 3$.

$\left(S_{2}\right) 2 \leq m_{j}$ and $m_{j} \neq 3$, for all $j<n$.

Figure 17 illustrates the lattice we obtain for $S=(4,4,2,2,2)$.

This result is not a direct generalization of Theorem 19, since we do not assume that $\Theta$ be the only nontrivial congruence of $K$. This additional condition is easy to accommodate:

Corollary. Let $S=\left(m_{j} \mid j<n\right)$ be a family of natural numbers, $n>1$. Then there is a finite sectionally complemented lattice $L$ with more than one element 


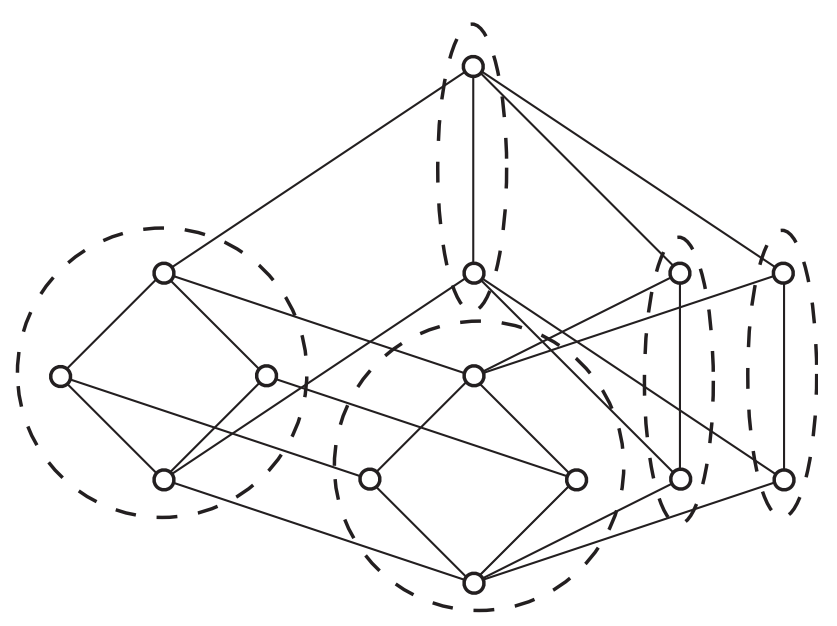

FIGURE 17. A lattice representing $S=(4,4,2,2,2)$.

with a unique nontrivial congruence $\Theta$ of $L$ such that $S$ is the spectrum of $\Theta$ iff $S$ satisfies $\left(S_{1}\right)$ and $\left(S_{2}\right)$, and additionally:

$\left(S_{3}\right) S$ is not constant, that is, there are $j, j^{\prime}<n$ satisfying that $m_{j} \neq m_{j^{\prime}}$.

$\left(S_{4}\right) n \neq 4$.

9.3. Valuation. There is a more sophisticated way of looking at spectra. Let $L$ be a finite lattice, and let $\Theta$ be a congruence of $L$. Then there is a natural map $v: L / \Theta \rightarrow \mathbb{N}$ (where $\mathbb{N}$ is the set of natural numbers) defined as follows: Let $a \in$ $L / \Theta$; then $a$ is a congruence class of $\Theta$, so we can define $v(a)=|a|$. We call $v$ a valuation on $L / \Theta$.

Now if $L$ is a finite sectionally complemented lattice and $\Theta$ is a nontrivial congruence of $L$, then we obtain the finite sectionally complemented lattice $K=L / \Theta$ and the valuation $v$ on $K$. The question is the following: Given a finite sectionally complemented lattice $K$ and a map $v: K \rightarrow \mathbb{N}$, when is $v$ a valuation?

Theorem 21 (G. Grätzer and E. T. Schmidt [48]). Let $K$ be a finite sectionally complemented lattice with more than one element, and let $v: K \rightarrow \mathbb{N}$. Then there exists a finite sectionally complemented lattice $L$ and a nontrivial congruence $\Theta$ of $L$, such that there is an isomorphism $\varphi: K \rightarrow L / \Theta$ satisfying

$$
v(a)=|\varphi(a)|, \quad \text { for all } a \in K,
$$

iff $v$ satisfies the following conditions:

$\left(V_{1}\right) v$ is anti-isotone, that is, if $a \leq b$ in $K$, then $v(a) \geq v(b)$ in $\mathbb{N}$.

$\left(V_{2}\right) 2 \leq v(a)$ and $v(a) \neq 3$, for all $a \in K$.

As a very small example, let us start with $K=M_{3}$ with a valuation as illustrated in Figure 18. The lattice $L$ we construct from this valuation is the one shown on Figure 17. 


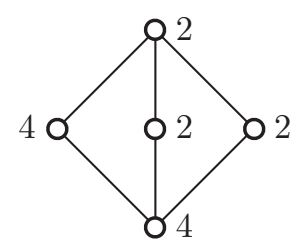

Figure 18. A valuation on $M_{3}$.

Again, we can ask about valuations induced by a finite sectionally complemented lattice $L$ and the unique nontrivial congruence $\Theta$ of $L$.

Corollary. Let $K$ be a finite sectionally complemented lattice with more than one element, and let $v: K \rightarrow \mathbb{N}$. Then there exists a finite sectionally complemented lattice $L$ and a unique nontrivial congruence $\Theta$ of $L$, such that there is an isomorphism $\varphi: K \rightarrow L / \Theta$ satisfying

$$
v(a)=|\varphi(a)|, \quad \text { for all } a \in K
$$

iff $v$ satisfies the conditions $\left(V_{1}\right)$ and $\left(V_{2}\right)$, and additionally, $v$ satisfies the following two conditions:

$\left(V_{3}\right) v$ is not a constant function.

$\left(V_{4}\right) K$ is simple.

\section{Simultaneous representations of two distributive lattices}

Let $L$ be a lattice and let $K$ be a sublattice of $L$. Then the restriction map

$$
\text { rs: } \operatorname{Con} L \rightarrow \operatorname{Con} K
$$

is a $\{0,1\}$-preserving meet-homomorphism. So we have two distributive lattices, Con $L$ and Con $K$, connected by a $\{0,1\}$-preserving meet-homomorphism. For a simultaneous representation of two distributive lattices, we are given two distributive lattices $D$ and $E$, a $\{0,1\}$-preserving meet-homomorphism $\varphi: D \rightarrow E$, and we ask when these can be represented as rs: Con $L \rightarrow$ Con $K$.

There is a growing literature of simultaneous representations of more than two distributive lattices interconnected by homomorphisms, called diagrams, discussed in detail in J. Tůma and F. Wehrung [78].

10.1. Restrictions as lattice homomorphisms. If $K$ is an ideal of $L$, then rs is a $\{0,1\}$-homomorphism of Con $L$ into Con $K$. In this case, for finite lattices, the problem of simultaneous representations was solved in G. Grätzer and H. Lakser [22]:

Theorem 22. Let $D$ and $E$ be finite distributive lattices; let $D$ have more than one element. Let $\varphi$ be a $\{0,1\}$-preserving homomorphism of $D$ into $E$. Then there exists a (sectionally complemented) finite lattice $L$ and an ideal $K$ of $L$ such that $D \cong \operatorname{Con} L, E \cong \operatorname{Con} K$, and $\varphi$ is represented by $\mathrm{rs}$, the restriction map. 

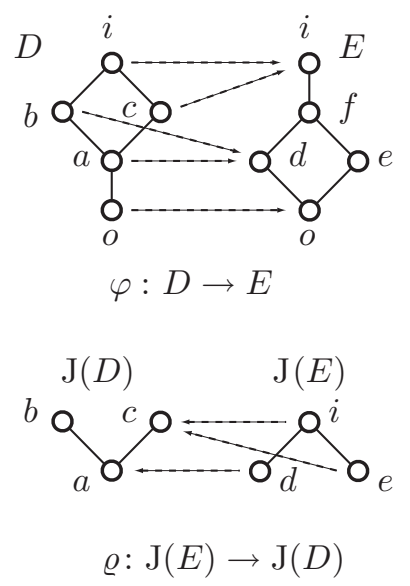

Figure 19. $\varphi: D \rightarrow E$ and $\varrho: \mathrm{J}(E) \rightarrow \mathrm{J}(D)$.

We outline the proof with a small example, as shown in Figure 19. We associate with the $\{0,1\}$-preserving homomorphism $\varphi$ an "inverse map" $\varrho: \mathrm{J}(E) \rightarrow \mathrm{J}(D)$, where $\varrho$ on an $x \in \mathrm{J}(E)$ is defined as the smallest element of $E$ that is mapped to an element $\geq x$ by $\varphi$.

Our plan is the following: We construct a chopped lattice $M$ as in Section 2.3 except that we triple, not double, every join-irreducible element, starting with the poset $\mathrm{J}(D) \cup \mathrm{J}(E)$. Every join-irreducible element $x$ has three copies, $x_{L}$ (left), $x_{M}$ (middle), and $x_{R}$ (right). We further double all these elements as in Section 2.3. So the basic building block is the chopped lattice of Figure 20, which already guarantees that $a_{L} \equiv 0$ is equivalent to $a_{M} \equiv 0$ is equivalent to $a_{R} \equiv 0$ is.

We take six building blocks: one for each element of $\mathrm{J}(D)$ and $\mathrm{J}(E)$.

We partially order $\mathrm{J}(D)$ and $\mathrm{J}(E)$ as in Section 2.3, using the middle elements. For instance, to achieve that $a \equiv 0$ implies that $b \equiv 0$, we insert $N\left(a_{M}, b_{M}\right)$, as illustrated in Figure 21.

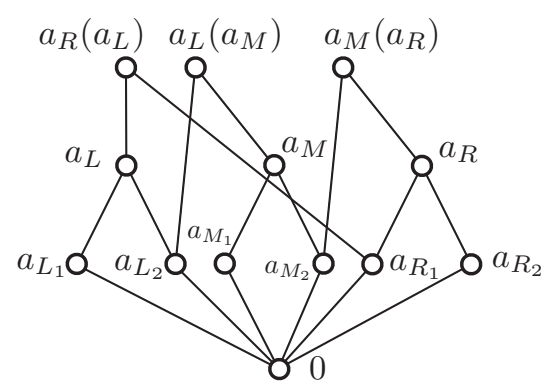

Figure 20. The basic building block. 


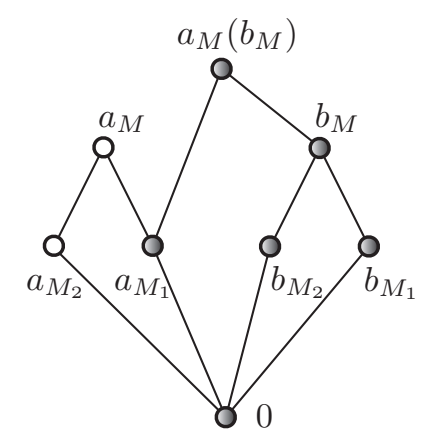

FIGURE 21. Inserting $N\left(a_{M}, b_{M}\right)$, the shaded elements.

Finally, we want to achieve that for $x \in \mathrm{J}(D), x \equiv 0$ is equivalent to $x \varrho \equiv 0$. One direction we code with the $L$-s, the other, with the $R$-s.

For an alternative proof, see E. T. Schmidt [71].

Problem 15. Is there an analogue of Theorem 22 for infinite lattices?

10.2. Extensions as join-homomorphisms. The "dual" of restriction is extension. Let $L$ be a lattice and let $K$ be a sublattice of $L$. Then the extension map:

$$
\mathrm{xt}: \text { Con } K \rightarrow \operatorname{Con} L
$$

maps a congruence $\Theta$ of $K$ to the congruence of $L$ generated by the subset $\Theta$ of $L^{2}$. The map xt is a 0-preserving join-homomorphism of Con $K$ into Con $L$. Obviously, xt separates 0 (a nontrivial congruence has a nontrivial extension).

More generally, let $K$ and $L$ be lattices, and let $\varphi$ be a homomorphism of $K$ into $L$. Then $\varphi$ induces a map xt $\varphi$ of $\operatorname{Con} K$ into $\operatorname{Con} L$ : For a congruence relation $\Theta$ of $K$, let the image $\Theta$ under xt $\varphi$ be the congruence relation of $L$ generated by the set $\Theta \varphi=\{\langle a \varphi, b \varphi\rangle \mid a \equiv b(\Theta)\}$.

One of the most persistent problems of lattice theory is the representation problem of distributive algebraic lattices as congruence lattice of lattices. A. P. Huhn in [59] and [60] attempted to solve this problem by simultaneous representation of finite distributive lattices as congruence lattices of finite lattices; see also P. Pudlák [65] and M. Tischendorf [76].

10.3. Huhn's result. The following result was proved by A. P. Huhn in [59] and [60] in the special case when $\psi$ is an embedding and was proved for arbitrary $\psi$ in G. Grätzer, H. Lakser, and E. T. Schmidt [26]:

Theorem 23. Let $D$ and $E$ be finite distributive lattices, and let

$$
\psi: D \rightarrow E
$$

be a 0-preserving join-homomorphism. Then there are finite lattices $K$ and $L, a$ lattice homomorphism $\varphi: K \rightarrow L$, and isomorphisms

$$
\alpha: D \rightarrow \operatorname{Con} K, \quad \beta: E \rightarrow \operatorname{Con} L
$$


with

$$
\psi \beta=\alpha(\mathrm{xt} \varphi) .
$$

Furthermore, $\varphi$ is an embedding iff $\psi$ separates 0 .

Theorem 23 concludes that the following diagram is commutative:

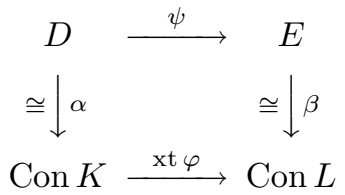

10.4. Fixing the base lattice. Theorem 23 claims the existence of the finite lattices $K$ and $L$. What if $K$ is given with Con $K \cong D$ ? This stronger version of Theorem 23 was proved in G. Grätzer, H. Lakser, and E. T. Schmidt [25]:

Theorem 24. Let $K$ be a finite lattice, let $E$ be a finite distributive lattice, and let $\psi: \operatorname{Con} K \rightarrow E$ be a 0-preserving join-homomorphism. Then there is a finite lattice $L$, a lattice homomorphism $\varphi: K \rightarrow L$, and an isomorphism $\beta: E \rightarrow$ Con $L$ with $\mathrm{xt} \varphi=\psi \beta$. Furthermore, $\varphi$ is an embedding iff $\psi$ separates 0 .

With an easy direct limit argument, Theorem 24 implies that every distributive algebraic lattice with countably many compact elements can be represented as the congruence lattice of a lattice, a result of Huhn's.

10.5. Isotone maps. rs is a meet-homomorphism and $x t$ is a join-homomorphism. What do we get by composing the two? Obviously, an isotone map. The converse was verified in G. Grätzer, H. Lakser, and E. T. Schmidt [26]:

Theorem 25. Let $D_{1}$ and $D_{2}$ be finite distributive lattices, and let

$$
\psi: D_{1} \rightarrow D_{2}
$$

be an isotone map. Then there are finite lattices $K_{1}, K_{2}$, L, a lattice embedding

$$
\varphi_{1}: K_{1} \rightarrow L
$$

a lattice homomorphism

$$
\varphi_{2}: K_{2} \rightarrow L
$$

and isomorphisms

$$
\alpha_{1}: D_{1} \rightarrow \operatorname{Con} K_{1}, \quad \alpha_{2}: D_{2} \rightarrow \operatorname{Con} K_{2}
$$

such that

$$
\alpha_{2} \circ \psi=\left(\operatorname{rs} \varphi_{2}\right) \circ\left(\operatorname{xt} \varphi_{1}\right) \circ \alpha_{1}
$$

that is, such that the diagram

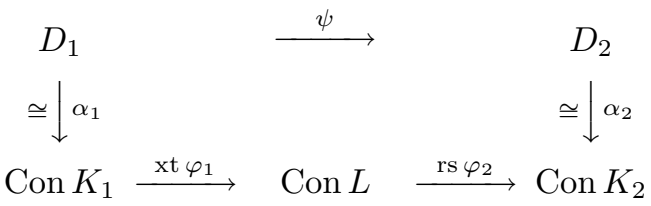

is commutative. Furthermore, $\varphi_{2}$ is an embedding iff $\psi$ preserves 0. 
We can again fix the base lattices, as verified in G. Grätzer, H. Lakser, and E. T. Schmidt [30]:

Theorem 26. Let $K_{1}$ and $K_{2}$ be arbitrary lattices with finite congruence lattices Con $K_{1}$ and Con $K_{2}$, respectively, and let

$$
\psi: \operatorname{Con} K_{1} \rightarrow \operatorname{Con} K_{2}
$$

be an isotone map. Then there is a lattice $L$ with finite congruence lattice, a lattice embedding

and a homomorphism

$$
\varphi_{1}: K_{1} \rightarrow L
$$

$$
\varphi_{2}: K_{2} \rightarrow L
$$

such that

$$
\psi=\left(\operatorname{rs} \varphi_{2}\right) \circ\left(\operatorname{xt} \varphi_{1}\right)
$$

Furthermore, $\varphi_{2}$ is an embedding iff $\psi$ preserves 0.

If $K_{1}$ and $K_{2}$ are finite, then $L$ can be chosen to be finite and atomistic.

In G. Grätzer, H. Lakser, and F. Wehrung [32], we found the following result with a remarkably simple proof:

Theorem 27. Let $K_{0}, K_{1}, K_{2}$ be lattices with finite congruence lattices and let $\eta_{1}: K_{0} \rightarrow K_{1}$ and $\eta_{2}: K_{0} \rightarrow K_{2}$ be lattice homomorphisms. Let $D$ be a finite distributive lattice, and, for $i \in\{1,2\}$, let $\psi_{i}$ : Con $K_{i} \rightarrow D$ be 0-preserving joinhomomorphisms such that

$$
\psi_{1} \circ \mathrm{xt} \eta_{1}=\psi_{2} \circ \mathrm{xt} \eta_{2} .
$$

There is then a lattice $L$, there are lattice homomorphisms $\varphi_{i}: K_{i} \rightarrow L$, for $i \in$ $\{1,2\}$, with

$$
\varphi_{1} \circ \eta_{1}=\varphi_{2} \circ \eta_{2}
$$

and there is an isomorphism $\alpha: \operatorname{Con} L \rightarrow D$ such that

$$
\alpha \circ \mathrm{xt} \varphi_{i}=\psi_{i}, \quad \text { for } i \in\{1,2\} .
$$

If $K_{1}$ and $K_{2}$ are finite, then $L$ can be chosen to be finite and atomistic.

This result enables us to prove Theorem 26 rather easily.

10.6. Huhn's theorem revisited. What can we say on the sizes of $K$ and $L$ in Theorem 23? Let $m$ and $n$ denote the number of join-irreducible elements of $D$ and $E$, respectively, and let $k=\max (m, n)$. The lattice $L$ constructed in Theorem 23 was of size $O\left(2^{2(n+m)}\right)$ and of breadth $n+m$.

In [29], G. Grätzer, H. Lakser, and E. T. Schmidt proved that $K$ and $L$ can be constructed as 'small' lattices of size $O\left(k^{5}\right)$ and of breadth three.

Theorem 28. Let $D$ be a finite distributive lattice with $n$ join-irreducible elements, let $E$ be a finite distributive lattice with $m$ join-irreducible elements, let $k=\max (m, n)$, and let

$$
\psi: D \rightarrow E
$$


be a 0-preserving join-homomorphism. Then there is a finite lattice $L$ of breadth 3 with $O\left(k^{5}\right)$ elements, a planar lattice $K$ with $O\left(n^{2}\right)$ elements, a lattice homomorphism $\varphi: K \rightarrow L$, and isomorphisms

$$
\alpha: E \rightarrow \operatorname{Con} L, \quad \beta: D \rightarrow \operatorname{Con} K
$$

with

$$
\psi \alpha=\beta(\operatorname{xt} \varphi),
$$

that is, such that the diagram

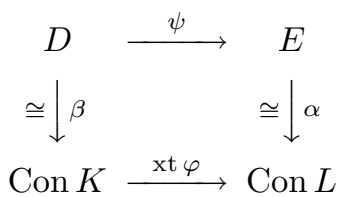

is commutative. Furthermore, $\varphi$ is an embedding iff $\psi$ separates 0 .

Problem 16. Is $O\left(k^{5}\right)$ the optimal size for the lattice $L$ ? Can one prove that size $O\left(k^{5}\right)$ cannot be replaced by size $O\left(k^{\alpha}\right)$, for any $\alpha<5$ ?

Problem 17. In Theorem 28, is breadth 3 optimal for $L$ ?

This is almost certainly so since a breadth 2 lattice cannot contain a $C_{2}^{3}$, making it very difficult to direct the congruences.

Problem 18. In Theorem 28, is the lattice $L$ of order dimension 3 ?

A. P. Huhn [57] introduced the 2-distributive identity:

$$
x \wedge\left(y_{1} \vee y_{2} \vee y_{3}\right)=\left(x \wedge\left(y_{1} \vee y_{2}\right)\right) \vee\left(x \wedge\left(y_{1} \vee y_{3}\right)\right) \vee\left(x \wedge\left(y_{2} \vee y_{3}\right)\right) .
$$

We call a lattice doubly 2-distributive, if satisfies the 2-distributive identity and its dual. In [40], we proved that in Theorem $28, K$ and $L$ can be constructed as doubly 2-distributive lattices.

Problem 19. In Theorem 24, if the lattice $K$ is (doubly) 2-distributive, can $L$ be constructed as a (doubly) 2-distributive lattice?

An affirmative answer would imply that every distributive algebraic lattice with countably many compact elements can be represented as the congruence lattice of a (doubly) 2-distributive lattice, a strenthening of Huhn's result mentioned at the end of Section 10.4.

\section{Beyond congruence-preserving extensions: Magic wands}

11.1. The two magic wand theorems. A typical way of constructing an algebra $A$ with a given congruence lattice $C$ is to construct an algebra $B$ with a much larger congruence lattice and then "collapsing" congruences $\Theta(a, b)$ and $\Theta(c, d)$ in $B$ in sufficient numbers so that the congruence lattice "shrinks" to $C$. To do this we need a "magic wand" that will make $a \equiv b$ equivalent to $c \equiv d$. Such a magic wand may be a pair of partial operations $f$ and $g$ such that $f(a)=c, f(b)=d$, and $g(c)=a, g(d)=b$. This is the start of the Congruence Lattice Characterization Theorem of Universal Algebras of the authors [35]. 
If you want to construct a lattice $L$ with a given congruence lattice $C$, how do you turn the action of the "magic wand" into lattice operations? To construct a simple modular lattice, E. T. Schmidt [69] started with the rational interval $K=[0,1]$ and by a "magic wand" he required that all $[a, b](0 \leq a<b \leq 1)$ satisfy that $a \equiv b$ be equivalent to $0 \equiv 1$. The action of the magic wand was realized with the extension $L=M_{3}[D]$, see Section 6.1 .

In G. Grätzer and E. T. Schmidt [46], we prove that one can apply the magic wand to arbitrary lattices with zero.

To set up "magic wands" for lattices formally, let $K$ be a bounded lattice, let $[a, b]$ and $[c, d]$ be intervals of $K$, and let $\varphi:[a, b] \rightarrow[c, d]$ be an isomorphism between these two intervals. We can consider $\varphi$ and $\varphi^{-1}$ as partial unary operations. Let us call a congruence $\Theta$ of $K$ a $\overleftrightarrow{\varphi}$-congruence iff $\Theta$ satisfies the Substitution Property with respect to the partial unary operations $\varphi$ and $\varphi^{-1}$. (The symbol $\leftrightarrow$ on top of $\varphi$ signifies that the partial operations go both ways.) Let $K_{\overleftrightarrow{\varphi}}$ denote the partial algebra obtained from $K$ by adding the partial operations $\varphi$ and $\varphi^{-1}$. Thus, a congruence relation of $K_{\overleftrightarrow{\varphi}}$ is the same as a $\overleftrightarrow{\varphi}$-congruence of $K$. We call $L$ a $\overleftrightarrow{\varphi}$ congruence-preserving extension of $K$, if a congruence of $K$ extends to $L$ iff it is a $\overleftrightarrow{\varphi}$-congruence and every $\overleftrightarrow{\varphi}$-congruence of $K$ has exactly one extension to $L$. As a special case, we get the concept of a congruence-preserving extension (in case, $\varphi$ is trivial).

Let us call $\varphi$ (resp., $\varphi^{-1}$ ) algebraic in $L$ iff there is a unary algebraic function $\mathbf{p}(x)$ (that is, $\mathbf{p}(x)$ is obtained from a lattice polynomial by substituting all but one variables by elements of $L$ ) such that $x \varphi=\mathbf{p}(x)$, for all $x \in[a, b]$ (resp., $x \varphi^{-1}=\mathbf{p}(x)$, for all $\left.x \in[c, d]\right)$.

Theorem 29. Let $K$ be a bounded lattice, let $[a, b]$ and $[c, d]$ be intervals of $K$, and let $\varphi:[a, b] \rightarrow[c, d]$ be an isomorphism between these two intervals. Then $K$ has $a \overleftrightarrow{\varphi}$-congruence-preserving extension into a bounded lattice $L$ such that both $\varphi$ and $\varphi^{-1}$ are algebraic in $L$. In particular, the congruence lattice of the partial algebra $K_{\leftrightarrow}$ is isomorphic to the congruence lattice of the bounded lattice $L$.

So the lattice $L$ constructed in this result is the magic wand for $\varphi$.

The realization of the magic wand is based on a construction of G. Grätzer and F. Wehrung [51] and an application of this construction in G. Grätzer and E. T. Schmidt [44].

In G. Grätzer, M. Greenberg, and E. T. Schmidt [20], a stronger result is proved. To state it, let $K$ be a bounded lattice, let $[a, b]$ and $[c, d]$ be intervals of $K$, let $\varphi$ be a homomorphism of $[a, b]$ onto $[c, d]$. We can consider, as above, $\varphi$ as a partial unary operation. We call a congruence $\Theta$ of $K$ a $\vec{\varphi}$-congruence iff $\Theta$ satisfies the Substitution Property with respect to the partial unary operation $\varphi$. Let $K_{\vec{\varphi}}$ denote the partial algebra obtained from $K$ by adding the partial operation $\varphi$. We call a lattice $L$ a $\vec{\varphi}$-congruence-preserving extension of $K$, if a congruence of $K$ extends to $L$ iff it is a $\vec{\varphi}$-congruence and every $\vec{\varphi}$-congruence of $K$ has exactly one extension to $L$. 
Theorem 30 (G. Grätzer, M. Greenberg, and E. T. Schmidt [20]). Let K be a bounded lattice, let $[a, b]$ and $[c, d]$ be intervals of $K$, and let $\varphi$ be a homomorphism of $[a, b]$ onto $[c, d]$. Then $K$ has a $\vec{\varphi}$-congruence-preserving extension into a bounded lattice $L$ such that $\varphi$ is algebraic in $L$. In particular, the congruence lattice of the partial algebra $K_{\vec{\varphi}}$ is isomorphic to the congruence lattice of the bounded lattice $L$. If $K$ is finite, then $L$ can be constructed as a finite lattice.

11.2. The construction. In Section 7.2, for a bounded lattice $K$, we introduced the boolean triple construction $\mathbf{M}_{3}\langle K\rangle$ of G. Grätzer and F. Wehrung [51].

For a congruence $\Theta$ of $K$, let $\Theta^{3}$ denote the congruence of $K^{3}$ defined componentwise. Let $\mathbf{M}_{3}\langle\Theta\rangle$ be the restriction of $\Theta^{3}$ to $\mathbf{M}_{3}\langle K\rangle$. Then $\mathbf{M}_{3}\langle\Theta\rangle$ is a congruence relation of $\mathbf{M}_{3}\langle K\rangle$ and every congruence of $\mathbf{M}_{3}\langle K\rangle$ is of the form $\mathbf{M}_{3}\langle\Theta\rangle$, for a unique congruence $\Theta$ of $K$.

In G. Grätzer and E. T. Schmidt [46], for $a, b \in L$ with $a<b$, the interval $\mathbf{M}_{3}\langle K, a, b\rangle$ of $\mathbf{M}_{3}\langle K\rangle$ was defined (the special case $\mathbf{M}_{3}\langle K, a\rangle=\mathbf{M}_{3}\langle K, a, 1\rangle$ was used in Section 7.2):

$$
\mathbf{M}_{3}\langle K, a, b\rangle=[\langle 0, a, 0\rangle,\langle 1, b, b\rangle] \subseteq \mathbf{M}_{3}\langle K\rangle .
$$

Again,

$$
\varphi_{a}: x \mapsto\langle x, a, x \wedge a\rangle
$$

is an embedding of $K$ into $\mathbf{M}_{3}\langle K, a, b\rangle$.

We shall use the notation:

$$
\begin{aligned}
I_{a, b} & =[\langle 0, a, 0\rangle,\langle 0, b, 0\rangle], \\
D_{a, b} & =[\langle 0, b, 0\rangle,\langle 1, b, b\rangle]=\{\langle x, b, x \wedge b\rangle \mid x \in K\} .
\end{aligned}
$$

Let the bounded lattice $K$, the intervals $[a, b]$ and $[c, d]$, and the isomorphism $\varphi:[a, b] \rightarrow[c, d]$ be given as in Theorem 29. We glue these lattices together as shown on Figure 23.

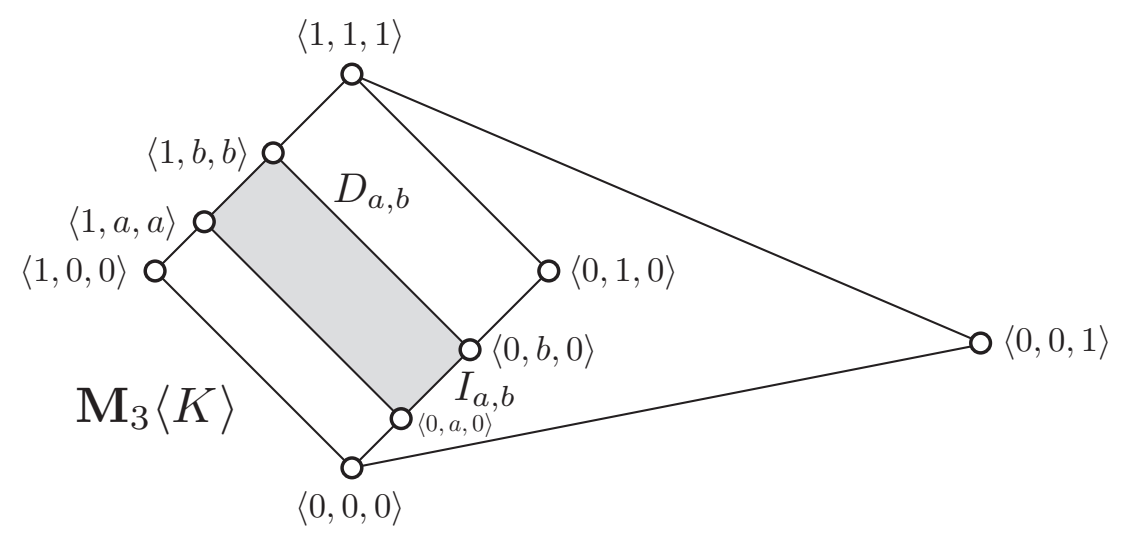

Figure 22. The lattice $\mathbf{M}_{3}\langle K\rangle$. The shaded area is $\mathbf{M}_{3}\langle K, a, b\rangle$. 


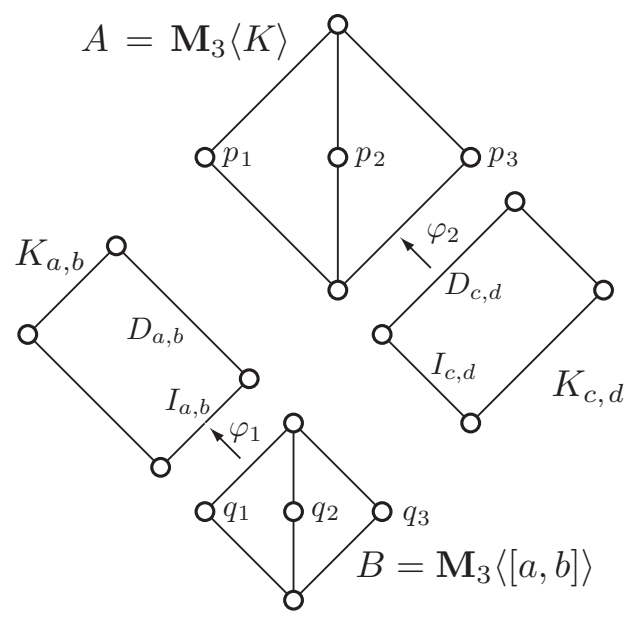

The four building blocks.

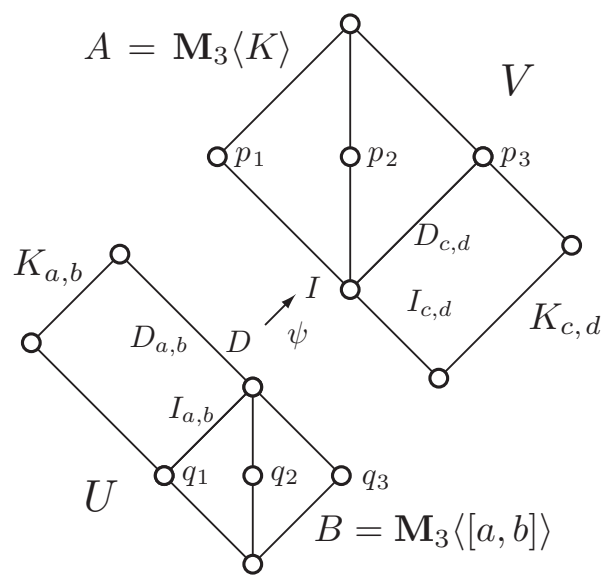

Two gluings.

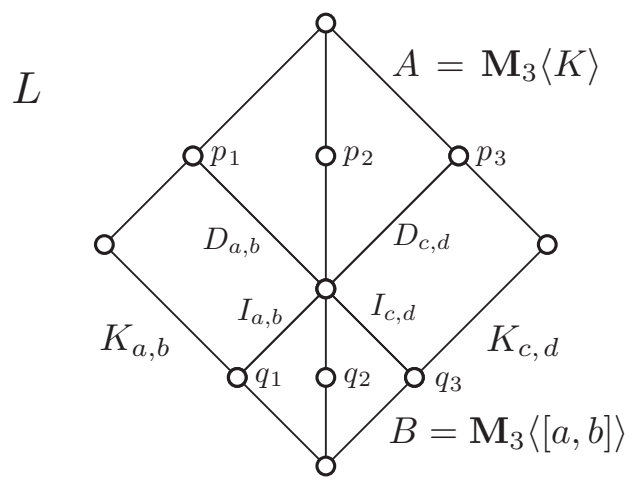

The final gluing.

Figure 23. Constructing the lattice $L$.

Problem 20. Let $K$ be a lattice, let $[a, b]$ and $[c, d]$ be intervals of $K$, and let $\varphi:[a, b] \rightarrow[c, d]$ be an isomorphism between these two intervals. Does $K$ have a $\overleftrightarrow{\varphi}$-congruence-preserving extension into a lattice $L$ such that both $\varphi$ and $\varphi^{-1}$ are algebraic in $L$ ?

Problem 21. Let $K$ be a bounded lattice, let $[a, b]$ and $[c, d]$ be intervals of $K$, and let $\varphi:[a, b] \rightarrow[c, d]$ be an isomorphism between these two intervals. Does $K$ have a $\overleftrightarrow{\varphi}$-congruence-preserving $\{0,1\}$-preserving extension into a lattice $L$ such that both $\varphi$ and $\varphi^{-1}$ are algebraic in $L$ ? 


\section{REFERENCES}

[1] J. Anderson and N. Kimura, The tensor product of semilattices, Semigroup Forum 16 (1968), 83-88.

[2] V.A. Baranskiü, On the independence of the automorphism group and the congruence lattice for lattices, Abstracts of lectures of the 15th All-Soviet Algebraic Conference, Krasnojarsk, July 1979, vol. 1, 11.

[3] Independence of lattices of congruences and groups of automorphisms of lattices (Russian), Izv. Vyssh. Uchebn. Zaved. Mat. 1984, no. 12, 12-17, 76. English translation: Soviet Math. (Iz. VUZ) 28 (1984), no. 12, 12-19.

[4] J. Berman, On the length of the congruence lattice of a lattice, Algebra Universalis 2 (1972), 18-19.

[5] G. Birkhoff, On groups of automorphisms, (Spanish) Rev. Un. Math. Argentina 11 (1946), $155-157$.

[6] K. P. Bogart, R. Freese, and J.P.S. Kung (editors), The Dilworth Theorems. Selected papers of Robert P. Dilworth, Birkhäuser Verlag, Basel-Boston, 1990, pp. 460-464.

[7] P. Crawley and R. P. Dilworth, Algebraic Theory of Lattices. Prentice-Hall, Englewood Cliffs, N. J., 1973.

[8] G. A. Fraser, The semilattice tensor product of distributive semilattices, Trans. Amer. Math. Soc. 217 (1976), 183-194.

[9] R. Freese, Congruence lattices of finitely generated modular lattices, Proceedings of the Ulm Lattice Theory Conference, pp. 62-70, Ulm, 1975.

[10] _ Computing congruence lattices of finite lattices, Proc. Amer. Math. Soc. (1997) 125, 3457-3463.

[11] R. Freese, J. Ježek, and J. B. Nation, Free lattices, Mathematical Surveys and Monographs, Vol. 42, American Mathematical Society, Providence, RI, 1995. viii+293 pp.

[12] R. Frucht, Herstellung von Graphen mit vorgegebener abstrakter Gruppe, Compos. Math. 6 (1938), 239-250.

[13] L Lattices with a given group of automorphisms, Canad. J. Math. 2 (1950), 417-419.

[14] N. Funayama and T. Nakayama, On the congruence relations on lattices, Proc. Imp. Acad. Tokyo 18 (1942), 530-531.

[15] G. Grätzer, Lattice Theory. First Concepts and Distributive Lattices, W. H. Freeman and Co., San Francisco, Calif., 1971. xv+212 pp.

[16] G. Grätzer, General Lattice Theory, second edition, new appendices by the author with B. A. Davey, R. Freese, B. Ganter, M. Greferath, P. Jipsen, H. A. Priestley, H. Rose, E. T. Schmidt, S. E. Schmidt, F. Wehrung, and R. Wille. Birkhäuser Verlag, Basel, 1998. xx+663 pp. ISBN: 0-12-295750-4, ISBN: 3-7643-5239-6.

[17] G. Grätzer, Congruence Lattices 101, ORDAL '96 (Ottawa, ON), Theoret. Comput. Sci. 217 (1999), 279-289.

[18] G. Grätzer and M. Greenberg, Lattice tensor products. I. Coordinatization, Acta Math. Hungar. 95 (4) (2002), 265-283.

[19] _ Lattice tensor products. IV. Infinite lattices, Submitted to Acta Math. Hungar.

[20] G. Grätzer, M. Greenberg, and E. T. Schmidt, Representing congruence lattices of lattices with partial unary operations as congruence lattices of lattices. II. Interval ordering, Submitted to J. Algebra.

[21] G. Grätzer and H. Lakser, Extension theorems on congruences of partial lattices, Notices Amer. Math. Soc. 15 (1968), 732, 785.

[22] Homomorphisms of distributive lattices as restrictions of congruences, Can. J. Math. 38 (1986), 1122-1134.

[23] _ Congruence lattices of planar lattices, Acta Math. Hungar. 60 (1992), pp. 251-268.

[24] G. Grätzer, H. Lakser, and E. T. Schmidt, Congruence lattices of small planar lattices, Proc. Amer. Math. Soc. 123 (1995), 2619-2623.

[25] _ Congruence representations of join-homomorphisms of distributive lattices: A short proof, Math. Slovaca 46 (1996), 363-369. 
[26] _ Isotone maps as maps of congruences. I. Abstract maps, Acta Math. Acad. Sci. Hungar. 75 (1997), 105-135.

[27] _ Congruence lattices of finite semimodular lattices, Canad. Math. Bull. 41 (1998), 290-297.

[28] , Restriction of standard congruences on lattices, Contributions to General Algebra, 10 (Klagenfurt, 1997), 167-175, Heyn, Klagenfurt, 1998.

[29] _ Congruence representations of join-homomorphisms of finite lattices: size and breadth, J.Austral Math. Soc. 68 (2000), 85-103.

[30] - Isotone maps as maps of congruences. II. Concrete maps, Acta Math. Acad. Sci. Hungar. 92 (2001), 233-238.

[31] G. Grätzer, H. Lakser, and R. W. Quackenbush, The structure of tensor products of semilattices with zero, Trans. Amer. Math. Soc. 267 (1981), 503-515.

[32] G. Grätzer, H. Lakser, and F. Wehrung, Congruence amalgamation of lattices, Acta Sci. Math. (Szeged) 66 (2000), 3-22.

[33] G. Grätzer, I. Rival, and N. Zaguia, Small representations of finite distributive lattices as congruence lattices, Proc. Amer. Math. Soc. 123 (1995), 1959-1961. Correction: 126 (1998), 2509-2510.

[34] G. Grätzer and E. T. Schmidt, On congruence lattices of lattices, Acta Math. Acad. Sci. Hungar. 13 (1962), 179-185.

[35] Characterizations of congruence lattices of abstract algebras, Acta Sci. Math. (Szeged) 24 (1963), 34-59.

[36] _ A lattice construction and congruence-preserving extensions, Acta Math. Hungar. 66 (1995), 275-288.

[37] _ Congruence lattices of p-algebras, Algebra Universalis 33 (1995), 470-477.

[38] _ The Strong Independence Theorem for automorphism groups and congruence lattices of finite lattices, Beiträge Algebra Geom. 36 (1995), 97-108.

[39] _ Complete congruence lattices of join-infinite distributive lattices, Algebra Universalis 37 (1997), 141-143.

[40] _ Representations of join-homomorphisms of distributive lattices with doubly 2-distributive lattices, Acta Sci. Math. (Szeged) 64 (1998), 373-387.

[41] _ Congruence-preserving extensions of finite lattices into sectionally complemented lattices, Proc. Amer. Math. Soc. 127 (1999), 1903-1915.

[42] _ Sublattices and standard congruences, Mailbox, Algebra Universalis 41 (1999), 151-153.

[43] - On finite automorphism groups of simple arguesian lattices, Studia Sci. Math. Hungar. 35 (1999), 247-258.

[44] _ Regular congruence-preserving extensions. Algebra Universalis 46 (2001), 119-130.

[45] Congruence-preserving extensions of finite lattices to semimodular lattices, Houston J. Math. 27 (2001)

[46] _ Representing congruence lattices of lattices with partial unary operations as congruence lattices of lattices. I. Interval equivalence. J. Algebra.

[47] _ On the Independence Theorem of related structures for modular (arguesian) lattices. Submitted to Studia Sci. Math. Hungar.

[48] _ Congruence class sizes in finite sectionally complemented lattices. Canadian Math. Bulletin.

[49] _ Finite lattices with isoform congruences. Manuscript.

[50] G. Grätzer, E. T. Schmidt, and K. Thomsen, Congruence lattices of uniform lattices. Houston J. Math.

[51] G. Grätzer and D. Wang, A lower bound for congruence representations, Order 14 (1997), 67-74.

[52] G. Grätzer and F. Wehrung, Proper congruence-preserving extensions of lattices, Acta Math. Hungar. 85 (1999), 175-185.

[53] __ A new lattice construction: the box product, J. Algebra 221 (1999), 315-344. 
[54] _ The Strong Independence Theorem for automorphism groups and congruence lattices of arbitrary lattices, Adv. in Appl. Math. 24 (2000), 181-221.

[55] A survey of tensor products and related constructions in two lectures, Algebra Universalis 45 (2001), 117-134.

[56] _ On the number of join-irreducibles in a congruence representation of a finite distributive lattice, Algebra Universalis.

[57] A. P. Huhn, Schwach distributive Verbände. I. Acta Sci. Math. (Szeged) 33 (1972), 297-305.

[58] _ Two notes on $n$-distributive lattices. Lattice theory (Proc. Colloq., Szeged, 1974), pp. 137-147. Colloq. Math. Soc. János Bolyai, Vol. 14, North-Holland, Amsterdam, 1976.

[59] - On the representation of distributive algebraic lattices, I. Acta Sci. Math. (Szeged) 45 (1983), 239-246.

[60] _ On the representation of distributive algebraic lattices. II, Acta Sci. Math. 53 (1989), 3-10.

[61]_ On the representation of distributive algebraic lattices. III, Acta Sci. Math. 53 (1989), 11-18.

[62] M. F. Janowitz, Section semicomplemented lattices, Math. Z. 108 (1968), 63-76.

[63] H. Lakser, The Tischendorf-Tima characterization of congruence lattices of lattices, manuscript, 1994.

[64] O. Ore, Theory of equivalence relations, Duke Math. J. 9 (1942), 573-627.

[65] P. Pudlák, On congruence lattices of lattices, Algebra Universalis 20 (1985), 96-114.

[66] P. Pudlák and J. Tůma, Every finite lattice can be embedded into a finite partition lattice, Algebra Universalis 10 (1980), 74-95.

[67] E. T. Schmidt, Über die Kongruenzverbänder der Verbände, Publ. Math. Debrecen 9 (1962), 243-256.

[68] Zur Charakterisierung der Kongruenzverbände der Verbände, Mat. Časopis Sloven. Akad. Vied. 18 (1968), 3-20.

[69] Every finite distributive lattice is the congruence lattice of some modular lattice, Algebra Universalis 4 (1974), 49-57.

[70] _ On the length of the congruence lattice of a lattice, Algebra Universalis 5 (1975), $98-100$.

[71] _ Congruence lattices of complemented modular lattices, Algebra Universalis 18 (1984), 386-395.

[72] _ Homomorphism of distributive lattices as restriction of congruences, Acta Sci. Math. (Szeged) 51 (1987), 209-215.

[73] _ Congruence lattices of modular lattices, Publ. Math. Debrecen 42 (1993), 129-134.

[74] S.-K. Teo, Representing finite lattices as complete congruence lattices of complete lattices, Ann. Univ. Sci. Budapest. Eötvös Sect. Math. 33 (1990), 177-182.

[75] $\quad$ On the length of the congruence lattice of a lattice, Period. Math. Hungar. 21 (1990), 179-186.

[76] M. Tischendorf, The representation problem for algebraic distributive lattices, $\mathrm{Ph}$. D. thesis, TH Darmstadt, 1992.

[77] M. Tischendorf and J. Tưma, The characterization of congruence lattices of lattices, manuscript, 1993.

[78] J. Tůma and F. Wehrung, A survey of recent results on congruence lattices of lattices, submitted to Algebra Universalis.

[79] A. Urquhart, A topological representation theory for lattices, Algebra Universalis 8 (1978), $45-58$.

[80] Y. Zhang, A note on "Small representations of finite distributive lattices as congruence lattices", Order 13 (1996), 365-367. 
Department of Mathematics, University of Manitoba, Winnipeg, MB R3T 2N2, Canada

E-mail address, George Grätzer: gratzer@cc.umanitoba.ca

URL, George Grätzer: http://server.math.umanitoba.ca/homepages/gratzer/

Mathematical Institute of the Budapest University of Technology and Economics, MÜEgYetem RKP. 3, H-1521 BudAPEST, Hungary

E-mail address, E.T. Schmidt: schmidt@math.bme.hu

URL, E. T. Schmidt: http://www.bme.math/ schmidt/ 